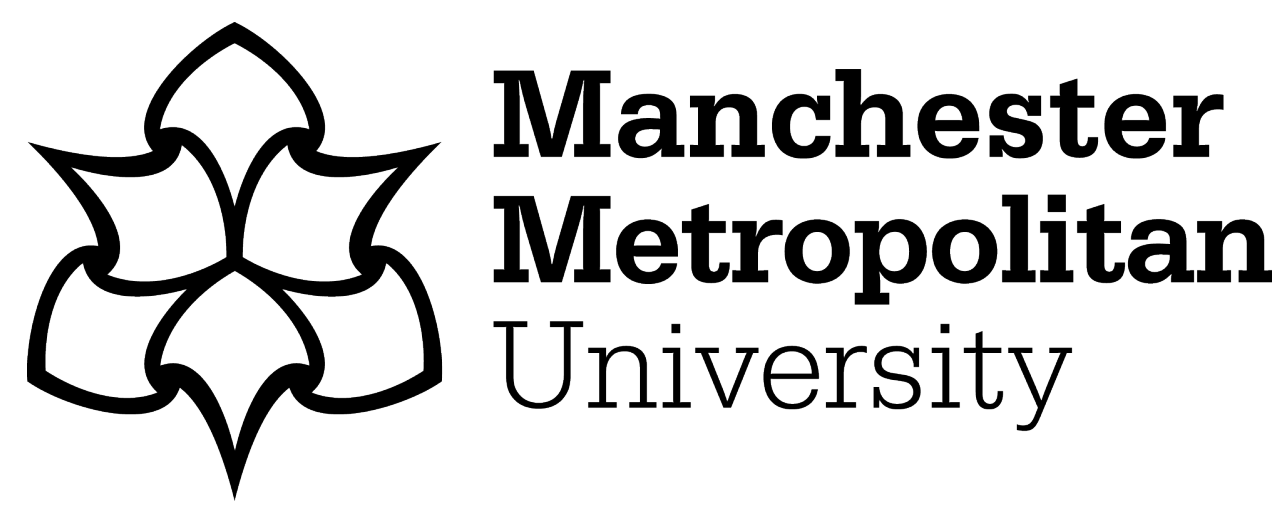

Martinez-Ferrer, Pedro J, Qian, Ling, Ma, Zhihua, Causon, Derek and Mingham, Clive (2018) An efficient finite-volume method to study the interaction of two-phase fluid flows with elastic structures. Journal of Fluids and Structures, 83. pp. 54-71. ISSN 0889-9746

Downloaded from: https://e-space.mmu.ac.uk/621424/

Version: Accepted Version

Publisher: Elsevier

DOI: https://doi.org/10.1016/j.jfluidstructs.2018.08.019

Usage rights: Creative Commons: Attribution-Noncommercial-No Derivative Works 4.0

Please cite the published version 


\title{
An efficient finite-volume method to study the interaction of two-phase fluid flows with elastic structures
}

\author{
Pedro J. Martínez-Ferrer ${ }^{\mathrm{a}, \mathrm{b}, *}$, Ling Qian ${ }^{\mathrm{b}}$, Zhihua $\mathrm{Ma}^{\mathrm{b}}$, Derek M. Causon ${ }^{\mathrm{b}}$, Clive G. Mingham ${ }^{\mathrm{b}}$ \\ ${ }^{a}$ Barcelona Supercomputing Center (BSC) \\ ${ }^{b}$ Centre for Mathematical Modelling and Flow Analysis, \\ Manchester Metropolitan University, \\ Chester Street, Manchester M1 5GD, United Kingdom
}

\begin{abstract}
We introduce a fluid-structure interaction (FSI) framework for the resolution of two-phase fluid flow problems and isothermal non-linear elastic bodies. The discretisation of both the fluid and solid governing equations relies exclusively on the finite volume method. A strong coupling partitioned approach is implemented to ensure the two-way coupling of information between the fluid and solid regions defined in the simulation. Moreover, this FSI framework is integrated on top of a multi-region coupling procedure developed in companion papers [Martínez-Ferrer et al. (2016, 2018)], which has been successfully applied in numerical wave tanks (NWTs), and shown to work transparently with standard domain decomposition techniques used in parallel simulations. Therefore, this retained approach results in a high performance computing strategy to carry out accurate and efficient FSI simulations of wave impacts against structures characteristic of ocean and coastal engineering problems.

We conduct a series of test cases to verify the implementation of this FSI framework and its parallel performance for an increasing number of CPU cores. These benchmarks include the dynamic and static responses of cantilever and clamped beams under various loads, a lid-driven flow in an elastic cavity, the water entry of an elastic wedge and, finally, a water dam impact on an elastic plate. The results obtained in this work agree well with analytical solutions, laboratory measurements as well as other numerical simulations reported in the literature.
\end{abstract}

Keywords: fluid structure interaction, two-phase fluid flow, coupling, hydroelasticity

\footnotetext{
* Corresponding author

Email address: pedro.martinez-ferrer@bsc.es (Pedro J. Martínez-Ferrer)
} 


\section{Introduction}

The interaction between two-phase fluid flows, e.g. waves, and structures is a complex problem that is gaining attention from the academic and industrial sectors due to its importance in offshore renewables worldwide [1, 2]. The harsh operating conditions, e.g. corrosion from exposure to seawater, high wind and wave loads, etc. to which these new energy systems are exposed can have a dramatic impact on both their survivability and long-term reliability. This is extremely important for offshore devices whose remote location requires helicopters and boats to carry out maintenance or even full replacement after stormy weather. Therefore, such systems must be designed more robustly in order to reduce their associated high maintenance costs. For this purpose, analysis and modelling tools can help by improving the performance and reliability of these devices so they can effectively contribute to the world energy production.

As the seas are often a hostile environment, the ability to model and predict waves loads is crucial for energy systems and compliant structures in ocean and offshore engineering such as catenary-moored floating vessels, articulated columns, guyed towers, tension leg platforms, etc. On the one hand, numerous experiments [3, 4] and simulations [5, 6] of waves impacting against rigid structures have already demonstrated that air pockets trapped in water bodies can be subject to expansion and compression by the surrounding liquid. As a result, such impacts can yield stronger peak pressures on the structure followed by pressure oscillations above and below atmospheric pressure. Although the aforementioned works do no take into account hydroelasticity effects, i.e. the structure is considered a solid rigid body, they offer valuable insight into how such pulsating loads can contribute to accelerating the ageing of offshore structures or even damage them permanently. On the other hand, hydroelastic effects produced by the impact of water bodies on simple geometries, e.g. wedges and plates, have been studied in the literature [7, 8, to determine their dynamic structural response. A recent experiment 9] carried out in both fully rigid and elastic sloshing wave tanks has found three different hydroelastic regimes during slamming. The first regime corresponds to a quasi-static deformation of the beam and the third regime is characterised by a free-vibration of the beam. During the second regime, a strong and fully coupled hydroelastic behaviour produces a rapid pressure increase that originates the maximum strain. As a consequence of the structural reaction and bending inwards, the hydrodynamic load further increases in comparison to the fully rigid experiment, which can compromise the integrity of the structure if a certain strain threshold is exceeded. The same authors found similar results using simplified theoretical models and numerical simulations [9]. Therefore, it seems that the solid rigid assumption is not always appropriate for the accurate modelling of waves interacting with structures and thus more complex flexible fluid-structure interaction models should be employed. 
From a numerical modelling point of view, the interaction between a flexible structure and its surrounding environment, e.g. waves and wind, can be decomposed into two main parts: (i) the modelling of the fluid medium with the aid of computational fluid dynamics (CFD) and (ii) the analysis of the structural response to its environment with the aid of computational structural mechanics (CSM). There is a wide variety of CFD methods for the resolution of free-surface flows. The finite volume method (FVM) remains the most popular, see for instance references [10,11, although numerous examples can be found in the literature using the boundary element method (BEM) [8] or the smoothed particle hydrodynamics (SPH) method [12, 13] to mention but a few. For the resolution of CSM problems, the finite element method (FEM) is widely established in both academia and industry [14, 15. To a much smaller extent, one can also find works that have demonstrated the capacity of the FVM to solve structural problems with large deformations [16, 17.

FSI simulations do require the coupling of CFD and CSM tools [18 and are commonly employed in ocean engineering problems [19]. FSI is part of a wide range of multi-physics problems involving different physical phenomena 20] such as fluid dynamics, solid mechanics, thermodynamics, radiation, magnetism, etc. Multiphysics simulations are rather complex and depend on the physical laws as well as the relevant time and spatial scales and therefore represent a challenging area for future research [21]. In the particular case of FSI simulations, there are several strategies for the coupling of CFD and CSM tools: some of them rely exclusively on the FEM [22, 23] or the FVM [24, 25] for both the resolution of the fluid and solid equations. However, the most popular methodology, and the one that has been widely adopted by the industry, consists of combining CFD software (typically based on the FVM) and CSM software (relying on the FEM) with a third-party multi-physics coupling manager [26, 27, 28, that often controls the data exchange between them through the computer file system (I/O level operations). Nevertheless, with the increasing demand on heterogeneous and high performance computing (HPC), most of these FSI strategies are no longer competitive due to their lack of support for parallel computing. For this reason, having the fluid and solid solvers integrated in the same executable code and using a parallel programming model yields efficient FSI simulations [29]. An efficient implementation for FSI can also be achieved via multi-region coupling [30, 31, which also offers the advantage of being completely transparent to parallel algorithms. Multi-region coupling applied to numerical wave tanks has demonstrated its potential to exploit HPC platforms when simulating large-scale ocean and naval engineering problems 30 .

The main objective of the present work is to develop a versatile and efficient FSI method integrated on the multi-region NWT framework "wsiFoam" [30] that relies exclusively on the FVM through the opensource library OpenFOAM [32]. The present FSI framework can therefore take advantage of HPC and, 
because it integrates the fluid and solid solvers as well as their coupling in one single executable file similarly to 29, it is a competitive and very efficient numerical tool for the study of typical wave-structure engineering problems often found in real-world engineering problems or in laboratory experiments. The remainder of this article is structured as follows. Section 2 describes the numerical procedures, i.e. the fluid dynamics and solid mechanics solvers as well as their coupling strategy. Section 3 presents different case studies and discusses the numerical results obtained. Finally, Section 4 is dedicated to conclusions and future work.

\section{Numerical implementation}

We implement our numerical procedures on top of the well-established CFD library OpenFOAM [32] relying on the FVM. This library is very popular among researchers and also industry and, because it is open-source, one can freely modify and improve it.

We integrate the fluid and solid mechanics solvers and the FSI coupling algorithm within our novel multi-region framework wsiFoam presented in the companion articles [30] and [31. In [30] wsiFoam was presented as a multi-region framework to couple incompressible and compressible two-phase flow solvers. Such a virtual wave-structure interaction (WSI) simulation environment allows coupling of an arbitrary number of incompressible and compressible two-phase fluid solvers in specific regions of a NWT in order to study aeration and compressibility effects characteristic of water-air mixtures. In 31 wsiFoam was further developed to deal with dynamic meshes in order to replicate physical moving paddles such as those commonly employed in experimental wave tanks. In that work it was proven that, by replicating the actual movement of physical paddles, (i) the generated waves were overall more accurate compared to other wave generation methods based on non-physical wave makers and (ii) the computational efficiency was similar, and in some cases even better, in comparison to the aforementioned methods thanks to the multi-region approach.

\subsection{Fluid solver}

For the sake of conciseness only the incompressible fluid solver of reference [30] is described here. This choice is also justified because compressibility effects are not important in the case studies presented in Section 3. This incompressible two-phase fluid solver is a modified version of the OpenFOAM solver "interFoam" 10] widely employed in naval and ocean engineering applications [33, 34. The two-phase flow mixture, composed of homogeneous air and water phases in mechanical equilibrium, is described by the means of the volume of fluid (VOF) method and an interface-capturing scheme that keeps a sharp free surface between the phases. 
The mass balance equation of the incompressible $(\boldsymbol{\nabla} \cdot \mathbf{U}=0)$ two-phase flow mixture can be reduced to the mass balance equation of the water volume fraction $\alpha$ (ratio of water volume to mesh cell volume):

$$
\frac{\partial \alpha}{\partial t}+\nabla \cdot \mathbf{U} \alpha+\nabla \cdot \mathbf{U}_{\mathrm{c}} \alpha(1-\alpha)=0
$$

where $\mathbf{U}$ is the mixture velocity vector and $\mathbf{U}_{\mathrm{c}}=\min [\mathbf{U}, \max (\mathbf{U})]$. The density of the fluid mixture is given by $\rho=\alpha \rho_{\mathrm{w}}+(1-\alpha) \rho_{\mathrm{a}}$ with constant partial densities $\rho_{\mathrm{a}}=1.226 \mathrm{~kg} / \mathrm{m}^{3}$ and $\rho_{\mathrm{w}}=999 \mathrm{~kg} / \mathrm{m}^{3}$ for the air and water phases, respectively 1 . The last term in eq. (1) is an artificial compression parameter that sharpens the water-air interface and guarantees bounded values of $\alpha \in[0,1]$ by using the MULES procedure [10, 35].

The momentum equation of the homogeneous fluid mixture is

$$
\frac{\partial \rho \mathbf{U}}{\partial t}+\nabla \cdot(\rho \mathbf{U U})=\boldsymbol{\nabla} \cdot(\mu \boldsymbol{\nabla} \mathbf{U})+\sigma \kappa \nabla \alpha-\mathbf{g} \cdot \mathbf{x} \nabla \rho-\nabla p_{\mathrm{d}}
$$

where $\sigma=0.07 \mathrm{~kg} / \mathrm{s}^{2}$ refers to the surface tension coefficient and $\kappa=\boldsymbol{\nabla} \cdot(\boldsymbol{\nabla} \alpha /|\nabla \alpha|)$ represents the curvature of the interface between the phases. The mixture viscosity is given by $\mu=\alpha \mu_{\mathrm{w}}+(1-\alpha) \mu_{\mathrm{a}}$ with constant partial viscosities $\mu_{\mathrm{a}}=1.47 \cdot 10^{-5} \mathrm{~kg} / \mathrm{ms}$ and $\mu_{\mathrm{w}}=1.11 \cdot 10^{-6} \mathrm{~kg} / \mathrm{ms}$. Finally, the dynamic pressure is derived from $p_{\mathrm{d}}=p-\rho \mathbf{g} \cdot \mathbf{x}$ where $|\mathbf{g}|=9.81 \mathrm{~m} / \mathrm{s}^{2}$ and $\mathbf{x}$ are the gravity and position vectors, respectively.

The governing equations (1)-(2) are linearised and then integrated over each control volume every time step to update the values of $\alpha$ and $\mathbf{U}$, respectively, and an additional pressure-corrector equation is solved for $p_{\mathrm{d}}[10]$. This solution procedure relies on the segregated projection algorithm PIMPLE [36], derived from the PISO procedure [37, which uses equation under-relaxation to guarantee solution convergence at each time step.

\footnotetext{
${ }^{1}$ http://www.mhtl.uwaterloo.ca/old/onlinetools/airprop/airprop.html
} 


\subsection{Solid solver}

The governing equation of an isothermal, non-linear elastic body can be expressed as

$$
\begin{aligned}
& \frac{\partial^{2} \rho_{\mathrm{s}} \mathbf{u}}{\partial t^{2}}=\boldsymbol{\nabla} \cdot \boldsymbol{\sigma}=\boldsymbol{\nabla} \cdot \underbrace{\left[\left(2 \mu_{\mathrm{s}}+\lambda_{\mathrm{s}}\right) \boldsymbol{\nabla} \mathbf{u}\right]}_{(\mathrm{I})}+\boldsymbol{\nabla} \cdot \underbrace{\left[\mu_{\mathrm{s}}(\boldsymbol{\nabla} \mathbf{u})^{\mathrm{T}}+\lambda_{\mathrm{s}} \mathbf{I} \operatorname{tr}(\boldsymbol{\nabla u})-\left(\mu_{\mathrm{s}}+\lambda_{\mathrm{s}}\right) \boldsymbol{\nabla u}\right]}_{\text {(II) }} \\
& +\boldsymbol{\nabla} \cdot \underbrace{\left[\mu_{\mathrm{s}} \boldsymbol{\nabla u} \cdot(\boldsymbol{\nabla u})^{\mathrm{T}}+\lambda_{\mathrm{s}} \mathbf{I} \operatorname{tr}\left(\boldsymbol{\nabla u} \cdot(\boldsymbol{\nabla u})^{\mathrm{T}}\right) / 2\right]}_{(\mathrm{III})} \\
& +\boldsymbol{\nabla} \cdot \underbrace{\left[\mu_{\mathrm{s}}\left(\boldsymbol{\nabla u}+(\boldsymbol{\nabla u})^{\mathrm{T}}+\boldsymbol{\nabla u} \cdot(\boldsymbol{\nabla u})^{\mathrm{T}}\right) \cdot \boldsymbol{\nabla u}\right]}_{(\mathrm{IV})} \\
& +\boldsymbol{\nabla} \cdot \underbrace{\left[\lambda_{\mathrm{s}} \mathbf{I} \operatorname{tr}\left(\boldsymbol{\nabla u}+(\boldsymbol{\nabla u})^{\mathrm{T}}+\boldsymbol{\nabla u} \cdot(\boldsymbol{\nabla u})^{\mathrm{T}}\right) \cdot \boldsymbol{\nabla u} / 2\right]}_{(\mathrm{V})},
\end{aligned}
$$

where $\boldsymbol{\sigma}=2 \mu_{\mathrm{s}} \boldsymbol{\varepsilon}+\lambda_{\mathrm{s}} \operatorname{tr}(\boldsymbol{\varepsilon}) \mathbf{I}$ is the second Piola-Kirchhoff stress tensor, $\boldsymbol{\varepsilon}=\left[\boldsymbol{\nabla u}+(\boldsymbol{\nabla u})^{\mathrm{T}}+\boldsymbol{\nabla u} \cdot(\boldsymbol{\nabla u})^{\mathrm{T}}\right] / 2$ is the Green-Lagrangian strain tensor and $\mathbf{I}$ is the unit tensor. The Lamé coefficients given by $\mu_{\mathrm{s}}=E /\left[2\left(1+\nu_{\mathrm{s}}\right)\right]$ and $\lambda_{\mathrm{s}}=\nu_{\mathrm{s}} E /\left[\left(1+\nu_{\mathrm{s}}\right)\left(1-2 \nu_{\mathrm{s}}\right)\right]$ are function of the Young's modulus $E$ and Poisson's ratio $\nu_{\mathrm{s}}$. Finally, $\rho_{\mathrm{s}}$ is the solid density and $\mathbf{u}$ the unknown solid displacement vector.

The FVM transforms Eq. (3) into a system of linear algebraic equations of the form $[\mathrm{A}] \cdot \mathbf{u}=\mathbf{b}$ for all the mesh cells. Coupling (II) and non-linear (III-V) terms of Eq. (3) form the vector $\mathbf{b}$ whilst the left hand side of Eq. (3) together with the Laplacian term (I) constitute the coefficient matrix [A]. This particular arrangement, where (I) is discretised implicitly and (II-V) are discretised explicitly, guarantees an equal coefficient matrix and improves the efficiency of the solution procedure [16]. Furthermore, because the right hand side of Eq. (3) depends on the unknown solid displacement vector $\mathbf{u}$, this equation needs to be solved iteratively, e.g. in a prediction-correction manner: $[\mathrm{A}]$ and $\mathbf{b}$ get updated with the value of $\mathbf{u}$ obtained from the previous iteration, which are then used to solve the system again. This process is repeated until a prescribed convergence value for $\mathbf{u}$ is reached.

The procedure described above is partially based on the one proposed in reference [25]. However, while we solve for the solid displacement $\mathbf{u}$ using the total Lagrangian formulation, the authors of [25] solved for the solid displacement increment $\delta \mathbf{u}$ using the updated Lagrangian formulation. Therefore, all the solid variables present in this work are referred to the original configuration of the system, i.e. $t=0 \mathrm{~s}$. The updated Lagrangian formulation, widely adopted by the vast majority of FEM structural solvers, needs to recompute solid variables and rotate the Cauchy incremental stresses every time step as the reference configuration changes over time. This results in significantly more calculations and, as a consequence, lower 
computational efficiency compared to the total Lagrangian formulation (see Section 3.1).

\subsection{FSI coupling procedure}

A strong coupling, partitioned, approach is retained for the FSI simulations carried out in this work. On the one hand, with the partitioned approach one needs to define independent fluid and solid mesh regions in which to solve Eqs. (1)-(2) and (3), respectively. Although this strategy usually leads to lower convergence properties compared to the unified, monolithic approach, it is relatively easier to implement and avoids forming ill-conditioned coefficient matrices that can potentially lead to numerical instabilities [38. On the other hand, in the partitioned approach kinematic and dynamic conditions need to be explicitly enforced at the fluid-solid interface thus requiring a strong coupling over the fluid and solid solvers to ensure best convergent results [18].

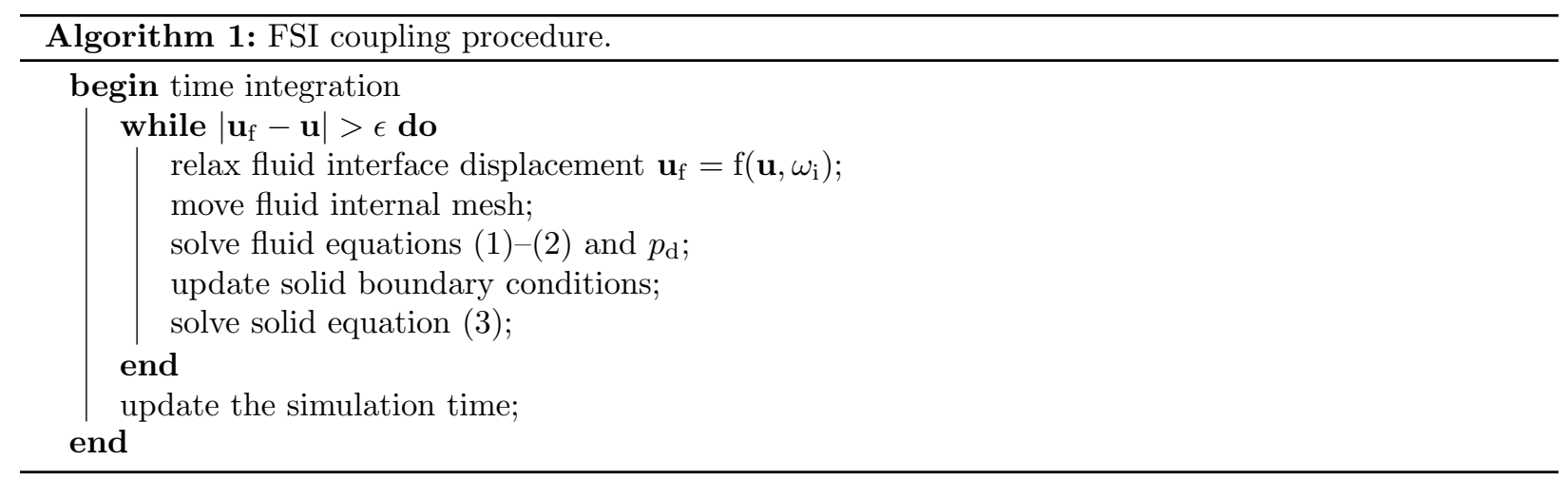

Algorithm 1 details the current FSI coupling procedure. The strong coupling approach imposes convergence of the fluid and solid displacements at their common interface. In order to speed up this iterative procedure, Aitken's method 39] is employed to relax the fluid interface displacement $\mathbf{u}_{\mathbf{f}}$ as a function of the solid displacement $\mathbf{u}$. This method provides faster convergence rates because it uses an adaptive relaxation factor $\omega_{\mathrm{i}}$ at each iteration $i$. Automated mesh motion [40, 41] is achieved by solving a Laplace equation to update the fluid internal mesh points using the values of $\mathbf{u}_{\mathrm{f}}$ as boundary conditions. Finally, the fluid equations (1)-(2) and $p_{\mathrm{d}}$ are solved in the new fluid mesh and pressure and viscous forces transferred to the structural solver, which uses them as boundary conditions to update the solid displacement $\mathbf{u}$ via Eq. (3). This process results in a two-way FSI coupling where information is exchanged in both directions and which is repeated until the desired convergence criteria is fulfilled.

In cases where the solid strain is small enough such that the geometry of the original computational domain is not altered significantly, Algorithm 1 can be simplified such that the fluid mesh remains completely 
static. In this configuration, pressure and viscous forces are still transferred from the fluid mesh to the solid mesh. However, the internal mesh points of the fluid computational domain no longer need to be recalculated. This results in the coupling information going in only one direction, i.e. fluid to solid, and consequently the outer iterative loop of Algorithm 1 is no longer necessary, reducing computational resources significantly. Herein, we refer to this procedure as one-way FSI coupling.

Pressure and viscous forces are transferred from the fluid mesh to the solid mesh through boundary conditions 30. For a given traction force $\boldsymbol{\tau}=\boldsymbol{\sigma} \cdot \mathbf{n}$, where $\mathbf{n}$ is the surface normal to the solid boundary, such a boundary condition can be written as

$$
\boldsymbol{\nabla} \mathbf{u} \cdot \mathbf{n}=\frac{\boldsymbol{\tau}-[(\mathrm{II})+(\mathrm{III})+(\mathrm{IV})+(\mathrm{V})] \cdot \mathbf{n}}{2 \mu_{\mathrm{s}}+\lambda_{\mathrm{s}}}
$$

The expression above is obtained from Eq. (3) by isolating the implicit term (I). Eq. (4) can be interpreted as a displacement gradient boundary, i.e. Neumann-type boundary condition, that can be solved for a prescribed traction force $\boldsymbol{\tau}$. The particular case in which $\boldsymbol{\tau}=\mathbf{0}$ is often referred to as traction free. In the case of FSI simulations, the traction force is a time-dependent function of the fluid viscous force and pressure $\boldsymbol{\tau}=\mu \boldsymbol{\nabla} \mathbf{U} \cdot \mathbf{n}-p \mathbf{n}$.

As previously mentioned, the fluid and solid solvers and the FSI coupling procedure are integrated in the multi-region framework wsiFoam [30, i.e. one single executable binary, which permits simulations of multiregion problems using an arbitrary number of fluid and solid solvers working together, and simultaneously, in different computational mesh regions. The type of solvers and the number of regions is decided by the user and usually depends on the degree of physics required or the size of the problem. For the sake of conciseness, the case studies presented herein are limited to one fluid region and one solid region.

\section{Results and discussions}

Unless otherwise stated, the following finite-volume discretisation schemes are used for the simulations carried out in this work: second-order linear spatial interpolation of flow and solid variables, second-order discretisation of spatial derivatives and second-order backward discretisation of time derivatives. Moreover, the two-way FSI coupling strategy is the default choice and a convergence criteria of $10^{-7}$ (which is a standard value in OpenFOAM) is fixed for all the iterative procedures. Finally, all the simulations and scalability tests presented below are executed on a Intel Core i7-6700 CPU with 4 physical cores (hyper-threading disabled). 

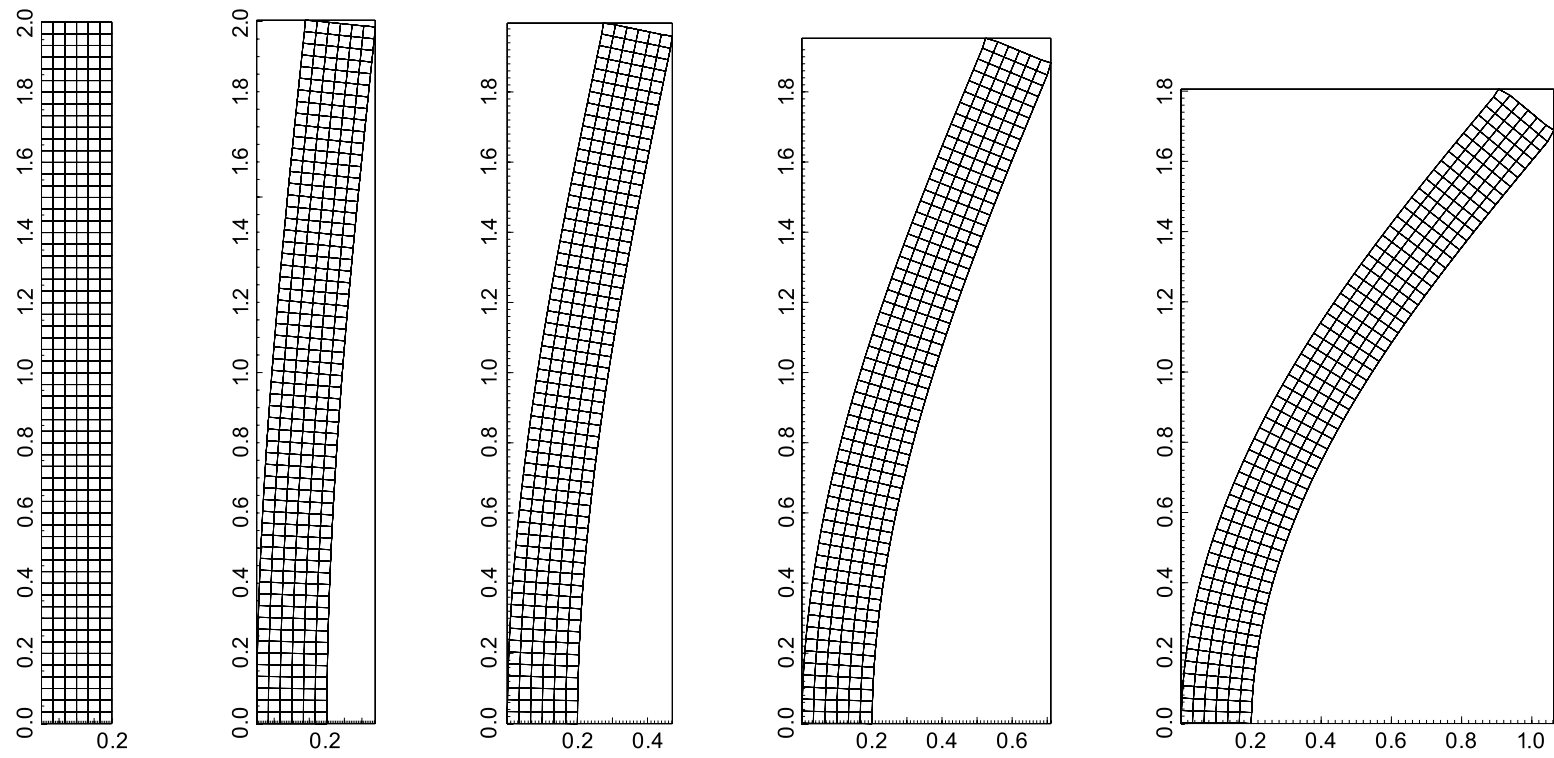

Figure 1: Deformation of a cantilever beam: computational mesh $(6 \times 60$ cells $)$ under loads $\phi=0, \phi=0.2, \phi=0.4, \phi=0.8$, $\phi=1.6$ from left to right. SI units.

\subsection{Deformation of cantilever and clamped beams}

The first benchmark used to validate our numerical tool consists of a cantilever beam under load, which is a classic structural problem that has been extensively studied analytically, experimentally and numerically. We are interested in both the dynamic response of the beam under a certain load as well as its static equilibrium shape.

This benchmark is based on the previous work of 25 where a cantilever beam of dimensions $0.2 \times 0.2 \times$ $2 \mathrm{~m}^{3}$, see Fig. 1, is subject to a sudden traction force applied at its top surface. The properties of the beam are as follows: density is $\rho_{\mathrm{s}}=1000 \mathrm{~kg} / \mathrm{m}^{3}$, Young's modulus $E=1.53 \times 10^{7} \mathrm{~Pa}$, Poisson's ratio $\nu_{\mathrm{s}}=0.3$, length $L=2 \mathrm{~m}$, cross-section area $A=0.04 \mathrm{~m}^{2}$ and the area moment of inertia about its neutral axis $I=1.33 \times 10^{-4} \mathrm{~m}^{4}$. The side walls of the beam share a traction free boundary condition and the bottom remains constrained with zero displacement. The top boundary has a parametric non-dimensional traction force $\phi=|\boldsymbol{\tau}| A L^{2} / E I$ (with $\boldsymbol{\tau}=|\boldsymbol{\tau}| \overrightarrow{\mathrm{i}}$ the surface traction vector measured in Pascal units). Henceforth two computational domains discretised with $6 \times 60 \times 1$ and $10 \times 100 \times 1$ mesh cells, respectively, are considered and a fixed time step of $2 \times 10^{-3} \mathrm{~s}$ [25] is chosen to carry out the simulations.

The transient response of the cantilever beam's tip displacement to different instantaneous traction forces applied at its top surface is illustrated in Fig. 2. The result is an oscillating movement of the tip around its static equilibrium, whose amplitude is close to the corresponding static displacement (see Table 1 ) and whose frequency is slightly smaller than the beam's first natural frequency of $1 \mathrm{~Hz}$ and increases with the 
(a)

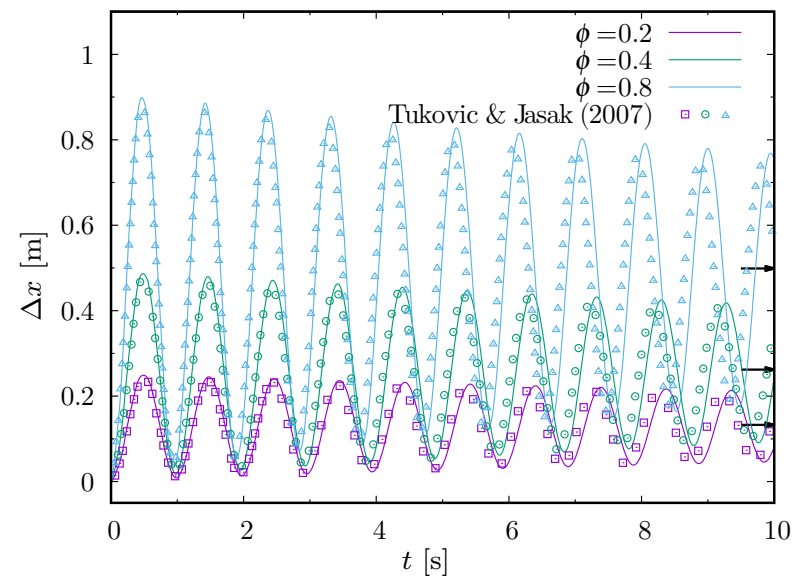

(b)

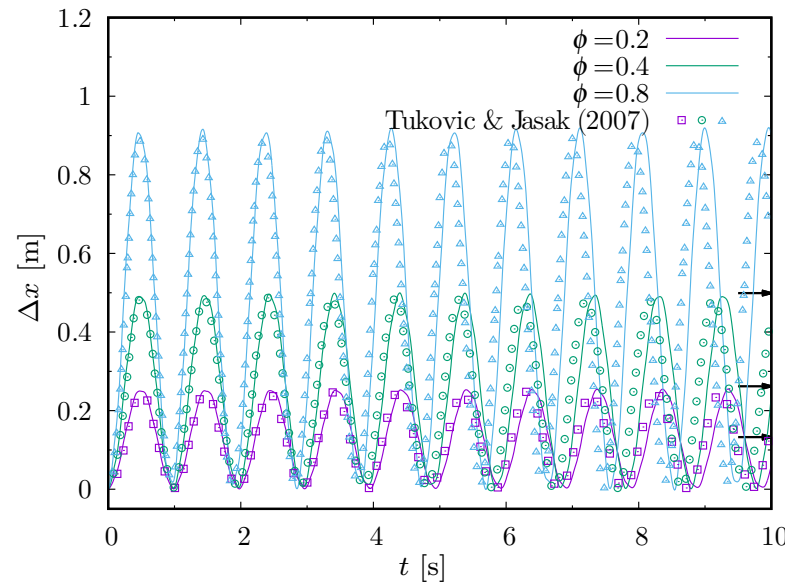

Figure 2: Deformation of a cantilever beam: time history of the beam's tip displacement for Euler (a) and backward (b) time discretisation schemes; arrows correspond to the steady state, beam's static tip displacement.

Table 1: Deformation of a cantilever beam: error $(\mathcal{E})$ and order of convergence $(s)$ of the beam's tip displacement under various loads $(\phi)$. The tip displacement $(d)$ is expressed in SI units.

\begin{tabular}{ccccccccc}
\hline$\phi$ & $d_{\text {ref }}$ & $d_{\mathrm{E}-\mathrm{B}}$ & $\mathcal{E}_{\mathrm{E}-\mathrm{B}}$ & $d_{6 \times 60}$ & $\mathcal{E}_{6 \times 60}$ & $d_{10 \times 100}$ & $\mathcal{E}_{10 \times 100}$ & $s_{6 \rightarrow 10}$ \\
\hline 0.2 & 0.1327 & 0.1333 & $0.46 \%$ & 0.1391 & $4.81 \%$ & 0.1289 & $2.89 \%$ & 0.9936 \\
0.4 & 0.2620 & 0.2667 & $1.80 \%$ & 0.2743 & $4.69 \%$ & 0.2546 & $2.81 \%$ & 1.0039 \\
0.8 & 0.4989 & 0.4989 & $6.90 \%$ & 0.5205 & $4.34 \%$ & 0.4864 & $2.50 \%$ & 1.0792 \\
1.6 & 0.8588 & 1.0667 & $24.2 \%$ & 0.8894 & $3.56 \%$ & 0.8447 & $1.65 \%$ & 1.5046 \\
\hline
\end{tabular}

vibration amplitude. It is worth noting that, when the first-order implicit Euler scheme is used for time discretisation, the amplitude is damped significantly over time due to excessive numerical diffusion, whilst it remains almost constant when the second-order backward scheme is employed, in agreement with Tukovic \& Jasak 25] albeit with a slight phase difference. Therefore, transient simulations based on this FVM based solver do require at least a second-order time discretisation scheme for accurate numerical prediction, whilst steady simulations can be carried out with first-order (or higher) accurate temporal schemes.

Moving on to the equivalent steady problem, Fig. 1 shows the cantilever beam's final deformation under various loads and Table 1 presents the numerical error and order of convergence corresponding to the beam's tip displacement. Such static equilibrium is easily achieved by adding a damping term of the form $\rho_{\mathrm{s}} K \partial \delta \mathbf{u} / \partial t$ with $K=10 \mathrm{~s}^{-1}$ to Eq. (3), which efficiently speeds the transient solution towards a steady state. Our results are compared against a reference, semi-analytical solution $d_{\text {ref }}$ obtained in reference [42], and against the analytical Euler-Bernoulli $(\mathrm{E}-\mathrm{B})$ beam theory solution, which reduces to $d_{\mathrm{E}-\mathrm{B}}=2 \phi / 3$ for this particular problem. As expected, the error of the Euler-Bernoulli solution increases for large deformations, i.e. moderate to large values of the non-dimensional traction force (see Fig. 1), and thus its applicability is 
Table 2: Deformation of a cantilever beam: averaged computation times and speed up obtained with updated (UL) and total Lagrangian (TL) formulations.

\begin{tabular}{cccc}
\hline Case & UL $(\mathrm{s})$ & TL $(\mathrm{s})$ & Speed up $(-)$ \\
\hline Transient & 752 & 542 & 1.39 \\
Steady & 433 & 56 & 7.73 \\
\hline
\end{tabular}

Table 3: Deformation of a clamped beam: error $(\mathcal{E})$ and order of convergence $(s)$ of the beam's centre of gravity displacement under various loads $(\phi)$. The tip displacement $(d)$ is expressed in SI units.

\begin{tabular}{cccccccccc}
\hline$\phi$ & $d_{\mathrm{E}-\mathrm{B}}$ & $d_{10 \times 100}$ & $\mathcal{E}_{10 \times 100}$ & $d_{16 \times 160}$ & $\mathcal{E}_{16 \times 160}$ & $d_{22 \times 220}$ & $\mathcal{E}_{22 \times 220}$ & $s_{10 \rightarrow 16}$ & $s_{10 \rightarrow 22}$ \\
\hline 0.01 & 0.00052 & 0.00057 & $8.63 \%$ & 0.00055 & $5.24 \%$ & 0.00054 & $4.09 \%$ & 1.1838 & 1.0847 \\
0.05 & 0.00260 & 0.00283 & $8.65 \%$ & 0.00274 & $5.25 \%$ & 0.00271 & $4.10 \%$ & 1.1829 & 1.0836 \\
0.10 & 0.00521 & 0.00566 & $8.63 \%$ & 0.00548 & $5.23 \%$ & 0.00542 & $4.08 \%$ & 1.1846 & 1.0856 \\
0.20 & 0.01042 & 0.01130 & $8.48 \%$ & 0.01095 & $5.10 \%$ & 0.01083 & $3.96 \%$ & 1.1982 & 1.1005 \\
0.40 & 0.02083 & 0.02245 & $7.78 \%$ & 0.02177 & $4.48 \%$ & 0.02153 & $3.36 \%$ & 1.2724 & 1.1826 \\
\hline
\end{tabular}

limited to $\phi \lesssim 4$. In all the configurations, the error $\mathcal{E}$ of our numerical solutions remain below $5 \%$ and the order of convergence, given by $s_{6 \rightarrow 10}=-\log \left|\mathcal{E}_{6 \times 60} / \mathcal{E}_{10 \times 100}\right| / \log (6 / 10)$, is smaller than 2 , which is consistent with the second-order discretisation schemes used for both spatial derivatives and spatial interpolations.

Table 2 reports the averaged computation times for the dynamic and static cases discussed above. Two formulations have been taken into consideration: total Lagrangian (TL, whose results are detailed above) and updated Lagrangian (UL, described in 25 and whose results are not presented here for the sake of conciseness). Using one single core, the TL configuration is almost 8 times faster than the UL counterpart for the steady problem and almost $40 \%$ faster for the transient case. As already mentioned, the total Lagrangian formulation has the advantage of requiring less calculations, which results in higher computational efficiency in this particular case.

Finally, we repeat the last numerical experiment on a clamped beam, which requires a slight readjustment of boundary conditions. In this new setup, the beam's bottom and top surfaces remain constrained to zero displacement, the right side wall remains traction free whilst the left side wall has the same non-dimensional traction force $\phi$ uniformly distributed along its surface. The rest of the parameters used in the simulation remain identical to the cantilever case. Our numerical results corresponding to the displacement of the beam's centre of gravity are presented in Table 3. The analytical Euler-Bernoulli beam theory solution for this particular problem becomes $d_{\mathrm{E}-\mathrm{B}}=2 \phi / 3840$ (about three orders of magnitude smaller than the cantilever solution) and is taken as the reference solution. Similarly to what is observed in the previous case, the numerical error increases for smaller displacement values and, in this particular case, it remains above $5 \%$ for a mesh composed of $10 \times 100$ cells and above $15 \%$ for the coarsest mesh with $6 \times 60$ cells (not shown 
(a)

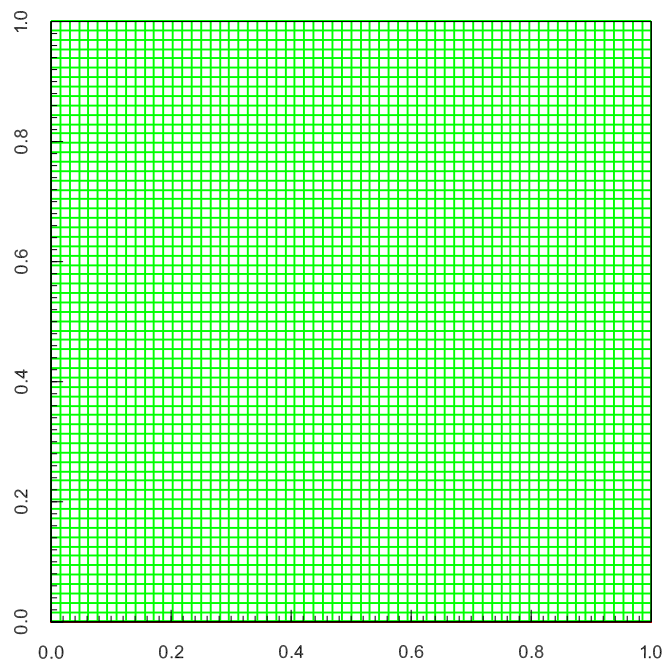

(b)

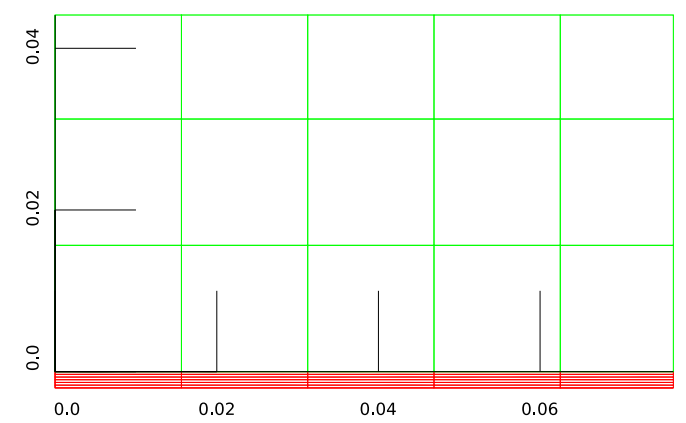

Figure 3: Lid-driven flow in an elastic cavity: (a) initial problem setup and (b) fluid (green) and solid (red) computational meshes zoomed in near the bottom-left corner. SI units.

in Table 3p. This benchmark indicates that finer meshes are therefore needed to capture smaller values of the solid displacement field. The orders of convergence, either $s_{10 \rightarrow 16}$ or $s_{10 \rightarrow 22}$, remain above unity, which is consistent with the spatial discretisation schemes employed. Finally, the averaged computation times associated with the four computational domains considered here, i.e. from $6 \times 60$ to $22 \times 220$ cells, were $18 \mathrm{~s}$, $33 \mathrm{~s}, 78 \mathrm{~s}$ and $168 \mathrm{~s}$, respectively.

\subsection{Lid-driven flow in an elastic cavity}

This problem has become a standard benchmark to validate numerical codes for fluid-structure interaction problems. It is also based on the classic benchmark "lid-driven cavity flow" that has been extensively used in the literature to validate fluid solvers. In this work, the problem setup is directly taken from reference 43 and consists of a square computational domain of $1 \mathrm{~m}$ side length, see Fig. 3.a). Contrarily to the classic lid-driven cavity flow benchmark, here the rigid bottom wall of the cavity is replaced by a flexible membrane with a thickness of $2 \mathrm{~mm}$ as depicted in Fig. 3. b). When an oscillating velocity is imposed at the top boundary, the recirculation of the fluid flow inside the cavity yields to the motion of the membrane.

It is worth mentioning that this benchmark deals with a single-phase fluid flow and hence we need to specify $\alpha=1$ everywhere in the computational domain, i.e. no free-surface, and thus the fluid properties become $\rho_{\mathrm{w}}=\rho=1 \mathrm{~kg} / \mathrm{m}^{3}$ and $\mu_{\mathrm{w}}=\mu=0.01 \mathrm{~kg} / \mathrm{ms}$. The density of the elastic membrane is $500 \mathrm{~kg} / \mathrm{m}^{3}, \mathrm{its}$ Young's modulus $250 \mathrm{~Pa}$ and the Poisson's ratio is equal to zero. An oscillating horizontal velocity of the form $u=1-\cos (2 \pi t / T) \mathrm{m} / \mathrm{s}$ with $T=5 \mathrm{~s}$ is specified at the top boundary of the computational domain. The 


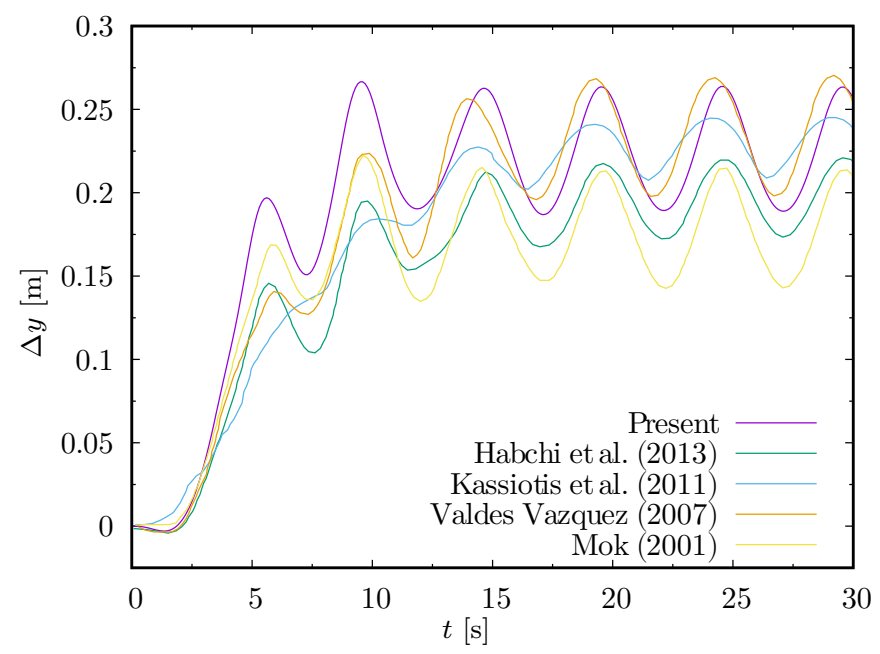

Figure 4: Lid-driven flow in an elastic cavity: time history of the elastic membrane's middle-point vertical displacement for various authors.

interface between the fluid and solid domains is treated as follows: on the one hand, the bottom boundary of the fluid domain is modelled as a non-slip wall; on the other hand, a traction boundary condition is specified at the top boundary of the solid domain, which gathers pressure and viscous forces from the fluid domain to reconstruct Eq. 44. The bottom boundary of the solid domain remains traction free whilst the left and right extremes share a zero displacement boundary condition. Following Valdes Vazquez (2007) [43], the rigid sidewalls of the cavity are treated as non-slip walls from the bottom up to $y=0.875 \mathrm{~m}$. From this ordinate upwards, a fixed pressure equal to zero is specified at the right wall (outlet) whilst a constant velocity slope $u=8(y-0.875) \mathrm{m} / \mathrm{s}$ is applied at the left wall (inlet). These boundary conditions are necessary for this fluid-structure interaction problem where (i) the incompressibility condition must be satisfied and (ii) the exact value of the pressure is required by the solid boundary conditions [44. The computational fluid and solid domains are discretised with $64 \times 64$ and $64 \times 6$ mesh cells, respectively, to guarantee converged results according to previous works 43, 45, 46. Finally, the simulation is carried out up to $30 \mathrm{~s}$ with a fixed time step of $10^{-2} \mathrm{~s} 43$ and the gravity force is not taken into consideration.

Fig. 4 shows the elastic membrane's middle-point vertical displacement obtained with the present FSI framework. Previous data from Mok (2001) [47, Valdes Vazquez (2007) [43, Kassiotis et al. (2010) [45] and Habchi et al. (2013) [46] are also included for a comparison between different approaches. Our present framework estimates the highest deformations during the transient phase and, after this period $(t=15 \mathrm{~s})$, our solution remains very close to the one of Valdes Vazquez albeit there exists a slight phase difference. Somewhat our solution is in phase with the data from Mok and Habchi et al. although these authors predicted 
a considerably smaller average displacement: $\overline{\Delta y} \approx 16 \mathrm{~cm}$ and $\overline{\Delta y} \approx 19 \mathrm{~cm}$, respectively, compared to the present value of $\overline{\Delta y} \approx 22.5 \mathrm{~cm}$. Despite the differences observed in the average displacement, all the solutions exhibit a similar trend after 20 seconds: an oscillating vertical displacement with a period of approximately $5 \mathrm{~s}$. These differences can be attributed to the various inlet and outlet boundary conditions specified by these authors to guarantee the incompressibility of the problem [44].

Finally, Fig. 5 shows a qualitative comparison between the present results and those obtained in reference [45. The transient period of both approaches is quite different and, as expected, our results show a larger displacement of the membrane during the transient phase. After $t=15 \mathrm{~s}$ the phase difference between the present solution and the one of Kassiotis et al. becomes evident in the side-by-side comparison although the periodicity is individually maintained on each approach, e.g. see $t=12.5 \mathrm{~s}$ vs. $t=22.5 \mathrm{~s}$ and $t=15 \mathrm{~s}$ vs. $t=25 \mathrm{~s}$.

The results presented above confirm that our numerical framework can be successfully applied to singlephase fluid flow FSI problems. The remainder of this paper seeks to assess the present framework on the resolution of two-phase fluid flow FSI problems and validates it against experimental data.

\subsection{Water entry of an elastic wedge}

The entry of wedges into water has been widely studied and documented in the literature. Such problem represents a canonical configuration that allows scientists to elaborate new theories and numerical models to reproduce the physics of water impacts. One example is the work of reference [8], where the assumptions of zero gravity and the wedge's constant speed allowed the authors to compare similarity, asymptotic and numerical solutions of a rigid wedge entering water. This classic fluid structure interaction problem was later extended to elastic wedges in [48, 49].

The present problem based on reference [48] is symmetric along the vertical axis and hence only half of it is considered: a two-dimensional computational domain of dimensions $0.95 \times 1.5 \mathrm{~m}^{2}$, see Fig. 6(a). The side of the wedge forms an angle of $30^{\circ}$ with respect to the calm water surface, as shown in Fig. 6(b), and measures $0.4 \mathrm{~m}$ and $0.2 \mathrm{~m}$ in the horizontal and vertical dimensions, respectively. Three values of wedge thickness are considered in this study: $5 \mathrm{~mm}, 8 \mathrm{~mm}$ and $11 \mathrm{~mm}$. This particular wedge behaves as a simplysupported beam, i.e. it is pinned at its two extremes, and bends as a consequence of penetrating the water surface at a constant speed of $1 \mathrm{~m} / \mathrm{s}$.

In the simulations carried out in this work, a static Euler reference frame is attached to the wedge resulting in the water coming upwards at a constant speed. Atmospheric pressure is set at the top and left boundaries of the fluid domain whilst a fixed velocity and symmetry conditions are imposed at the bottom 

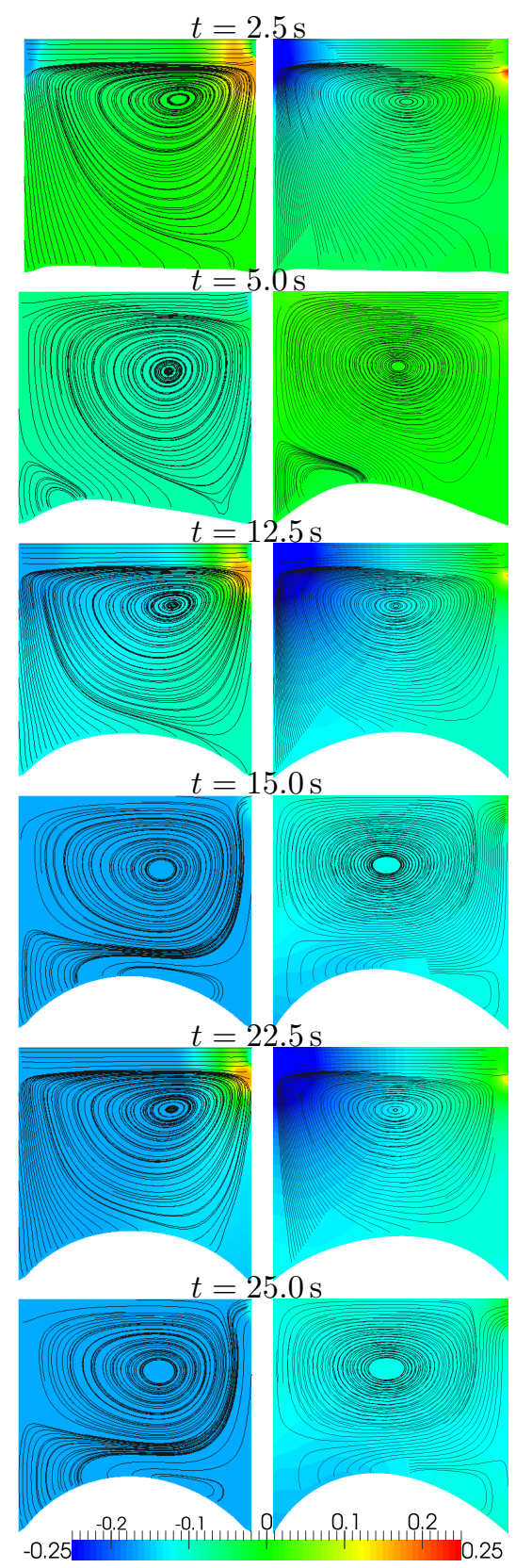

Figure 5: Lid-driven flow in an elastic cavity: snapshots of streamlines superposed to pressure contours of Kassiotis et al. (left) and the present numerical simulations (right) taken at different times. SI units. 
(a)

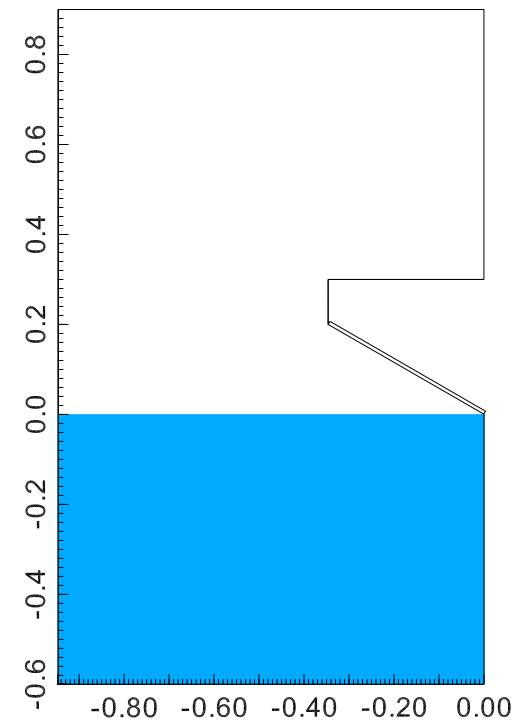

(b)

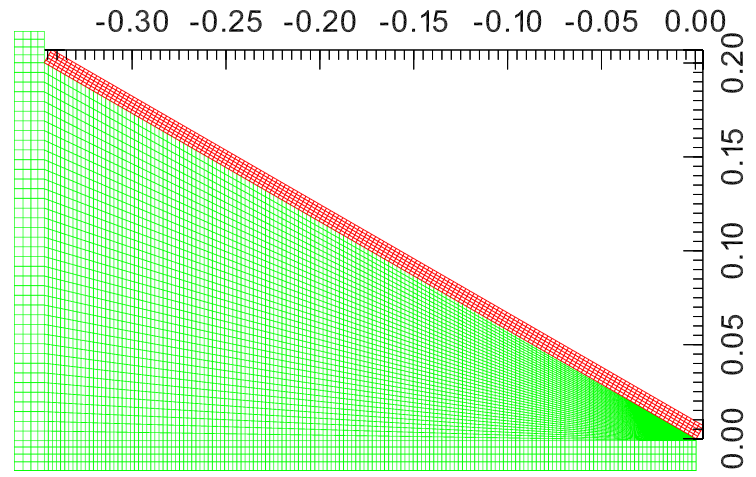

Figure 6: Water entry of an elastic wedge: (a) initial problem setup and (b) fluid (green) and solid (red) computational meshes near the wedge. SI units.

and right fluid boundaries, respectively. The solid domain representing the wedge consists of a deformable rectangle with variable thickness. On the one hand, the top boundary remains traction free whilst the bottom boundary has an imposed non-slip, traction boundary condition. On the other hand, the left and right extremes of the solid domain share a zero displacement boundary condition. It is worth noting that such boundary conditions correspond indeed to a clamped beam (also known as a built-in beam) instead of a beam pinned at its two extremes.

When solving the non-linear elastic solid equation (3) only two types of boundary conditions (4) can be imposed: fixed traction or fixed displacement. However, for a simply-supported beam it is necessary to define pinned boundary conditions to guarantee free rotation. Most commercial CSM software relying on the FEM have specialised beam packages to achieve this: they define a special type of "beam elements" where both nodal displacements and rotations are used to describe the structural deformation. Other commercial software tackle this via "rigid connectors" to produce the same effect. Lu et al. [48] employed beam elements whilst more recent works [50] simplify the structural analysis by solving the one-dimensional Euler-Bernoulli beam equation.

As neither beam elements nor rigid connectors are used in the present work, we decide to model the wedge as a clamped beam and compare our results against previous data based on simply-supported wedges. According to the Euler-Bernoulli beam theory, for (i) small deformations and (ii) uniform loads $\phi$, the 
(a)

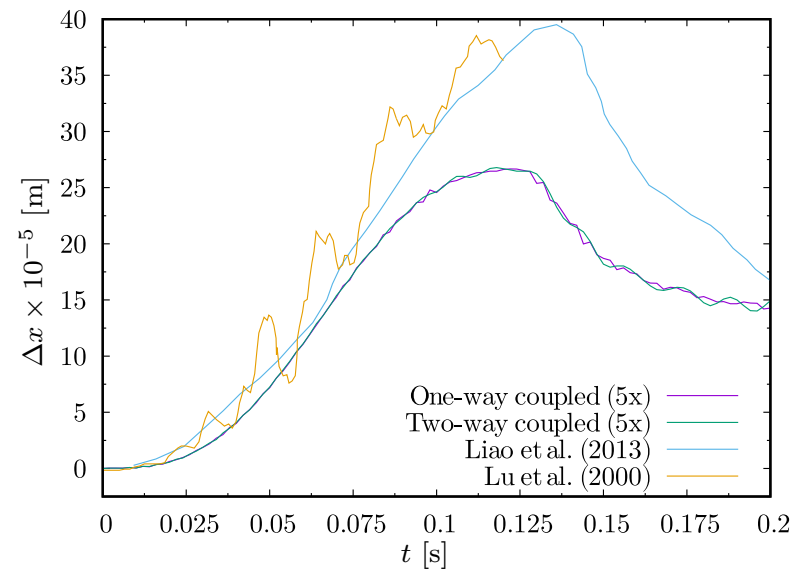

(b)

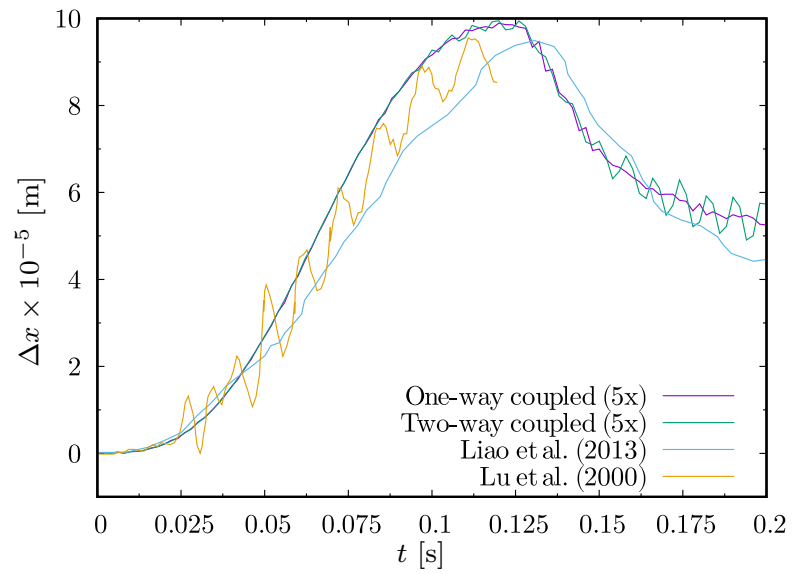

(c)

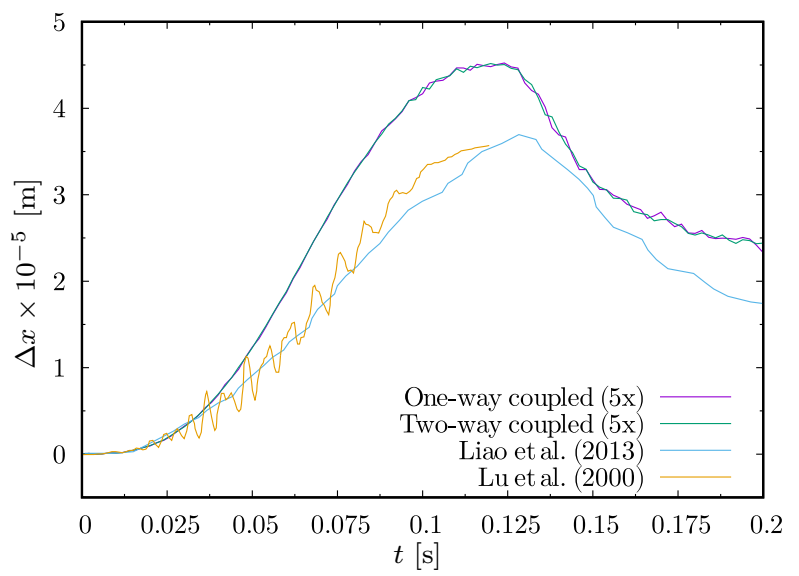

Figure 7: Water entry of an elastic wedge: wedge's middle point displacement for (a) $5 \mathrm{~mm}$, (b) $8 \mathrm{~mm}$ and (c) $11 \mathrm{~mm}$ thicknesses.

middle-point displacement of a simply-supported beam $\left(d=5 \phi L^{4} / 384 E I\right)$ is exactly 5 times larger than the equivalent clamped beam $\left(d=\phi L^{4} / 384 E I\right)$. For this particular problem, while the first condition holds, the load distribution is clearly not uniform and changes over time. Therefore, we can expect non-negligible differences using this approach.

The present simulations account for a total number of 15307 and 795 cells for the fluid and solid meshes, respectively, which guarantees converged solutions based on our previous work conducted on a rigid wedge under the same conditions [51. The wedge properties are as follows: density is $\rho_{\mathrm{s}}=7800 \mathrm{~kg} / \mathrm{m}^{3}$, Young's modulus $E=2 \times 10^{11} \mathrm{~Pa}$ and Poisson's ratio $\nu_{\mathrm{s}}=0.3$. Finally, the simulation is carried out up to $0.2 \mathrm{~s}$ with a fixed time step of $10^{-5} \mathrm{~s}[51$ and, following [8], gravity terms are neglected.

Fig. 7 shows the time history of the wedge's bottom middle point deflection for various thicknesses. 
(a)

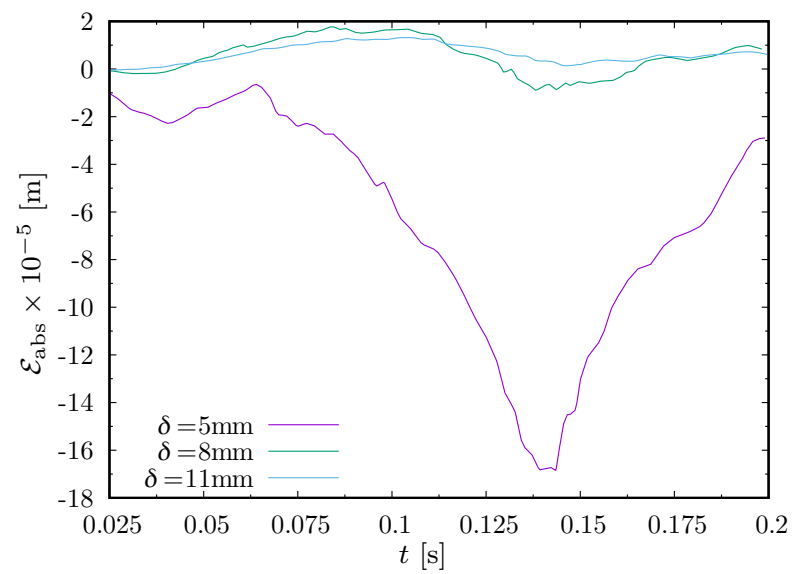

(b)

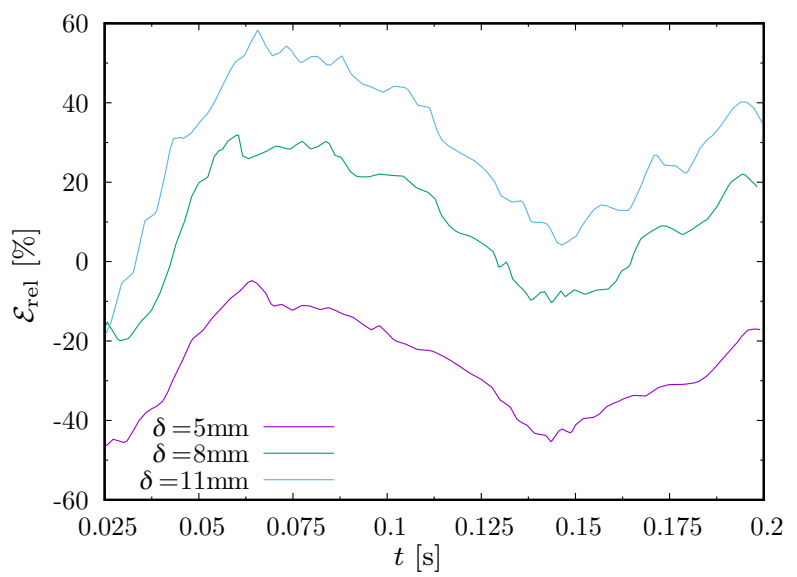

Figure 8: Water entry of an elastic wedge: (a) absolute and (b) relative error between the present numerical solution (one-way coupling) and the one from Liao et al.

Previous data reported by references [48, 49] are compared against our numerical results for one- and twoway coupling FSI strategies. We emphasise that our results based on a built-in beam are artificially multiplied by a factor of 5 in order to render them comparable against data reported on simply-supported beams. On the one hand, although wsiFoam solutions show a similar trend compared to previous data available in the literature, the deflection is clearly underpredicted for $\delta=5 \mathrm{~mm}$ and overpredicted for $\delta=11 \mathrm{~mm}$, see Figure 8. Indeed, the largest absolute discrepancy is found when $\delta=5 \mathrm{~mm}$ whilst the relative discrepancy remains bounded between $-40 \%$ and $+60 \%$, approximately. On the other hand, the present one- and twoway coupled solutions remain very close to each other. Small oscillations can be observed on the two-way FSI coupled simulation for $\delta=8 \mathrm{~mm}$ but remain significantly lower than those obtained in [48]. Overall, our numerical results are reasonably close to previous data after taking into consideration the simplified hypothesis that we have introduced.

Table 4 reports on the computation time measured in seconds needed to carry out each simulation. It can be readily seen the advantage of using the one-way FSI coupling strategy to speed up the simulations. As expected the computation runs significantly faster, by a factor above 30, compared to the two-way FSI coupling strategy. Finally, Table 5 reports on the averaged computation times and speed up of the FSI simulations for an increasing number of cores. For one-way coupling simulations the corresponding parallel speed up is almost 2 with 4 CPU cores (parallel performance around 50\%) and improves slightly using the two-way coupling strategy. These relatively low figures come from OpenFOAM's parallel implementation, based exclusively on the MPI protocol, which is known to suffer from some performance regressions [52]. 
Table 4: Water entry of an elastic wedge: computation times and speed up for one- and two-way coupled FSI simulations.

\begin{tabular}{cccc}
\hline$\delta(\mathrm{mm})$ & One-way $(\mathrm{s})$ & Two-way $(\mathrm{s})$ & Speed up $(-)$ \\
\hline 5 & 2842 & 104187 & 36.66 \\
8 & 2741 & 92013 & 33.57 \\
11 & 2643 & 86913 & 32.88 \\
\hline
\end{tabular}

Table 5: Water entry of an elastic wedge: averaged computation times and speedup for an increasing number of cores.

\begin{tabular}{cccccc}
\hline Coupling & 1 core $(\mathrm{s})$ & 2 cores $(\mathrm{s})$ & Speed up $(-)$ & 4 cores $(\mathrm{s})$ & Speed up $(-)$ \\
\hline One-way & 2742 & 1703 & 1.61 & 1401 & 1.96 \\
Two-way & 94371 & 50662 & 1.86 & 36019 & 2.62 \\
\hline
\end{tabular}

As a rule of thumb, around $10^{4}$ mesh cells per CPU core are required for good parallel scalability and thus greater parallel performance can be expected in much larger computations.

\subsection{Water dam impact on an elastic plate}

Similarly to the previous problem, the scientific literature is replete of experiments and numerical studies on water columns hitting rigid obstacles [53, 54]. These studies concentrate only on the fluid characteristics during the impact without considering any hydroelasticity effects that can increase or dampen the load pressure on the structure [9]. In this section we replicate the experiment presented in [55, which proposes a water dam impacting on an elastic plate in order to study hydroelasticity effects. Due to the severe deformation experienced by the plate, this particular test case becomes an excellent benchmark to assess the robustness of FSI numerical frameworks.

Fig. 9 (a) shows the three-dimensional computational domain of dimensions $0.8 \times 0.6 \times 0.2 \mathrm{~m}^{3}$ and the elastic plate located at the right hand side of the water tank at $0.596 \mathrm{~m}$. The plate dimensions are $0.004 \times$ $0.09 \times 0.2 \mathrm{~m}^{3}$ and its distance to the right wall of the tank is $0.2 \mathrm{~m}$, see Fig. 9 (b). The initial water column has a fixed width of $0.2 \mathrm{~m}$ and up to three different height values $H$ are considered: $0.2 \mathrm{~m}, 0.3 \mathrm{~m}$ and $0.4 \mathrm{~m}$. Once the gate that retains the column of water opens, the dam collapses under gravity and flows to the right hand side of the tank impacting on the elastic plate, which begins to bend.

The density of the plate is $1161.54 \mathrm{~kg} / \mathrm{m}^{3}$, its Young's modulus $3.5 \times 10^{6} \mathrm{~Pa}$ and the Poisson's ratio 0.48 . The walls of the tank and the plate share a non-slip condition whilst the tank's top boundary remains open to the atmosphere; additionally, traction boundary conditions are specified on the plate walls and, at its bottom, the displacement is fixed to zero (similar to a cantilever beam). The gravity term $\rho_{\mathrm{s}} \mathbf{g}$ is also added as an external force to the structural solver. The computational domain is discretised with 22270 and 88 cells for the fluid and solid meshes, respectively, and the simulation is carried out up to $0.5 \mathrm{~s}$ with a fixed 
(a)

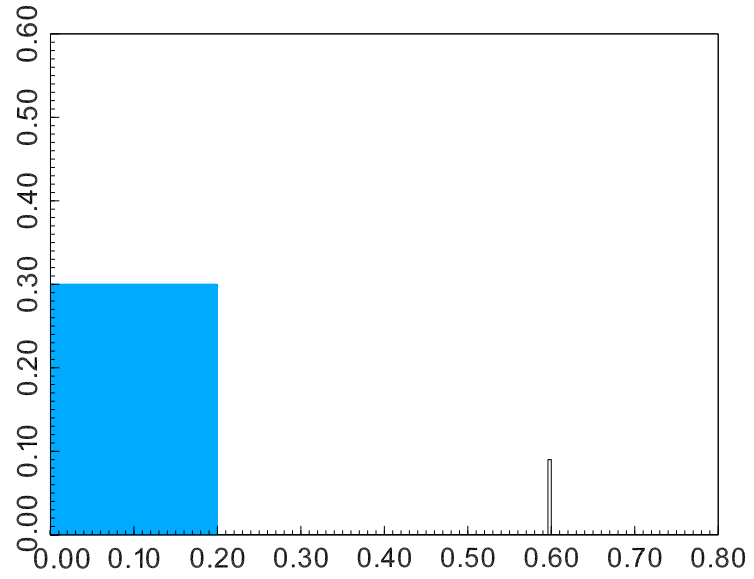

(b)

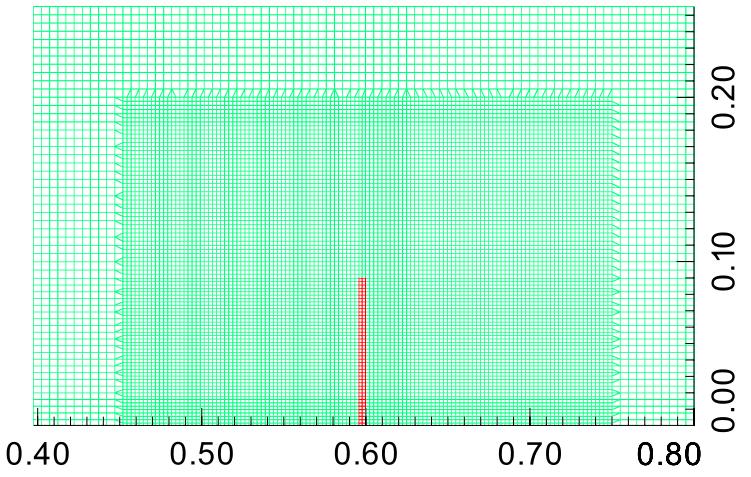

Figure 9: Water dam impact on an elastic plate: (a) initial problem setup $(H=0.3 \mathrm{~m})$ and (b) fluid (green) and solid (red) computational meshes near the plate. SI units.

time step of $10^{-4} \mathrm{~s}[51$.

Fig. 10 shows the horizontal displacement of the tip of the elastic plate as a function of the time for three different water column heights. Our numerical results are in good agreement with those obtained in reference [55] and also capture the negative displacements (small bump on the figure) due to the initial impact of the water front against the bottom of the elastic plate. It can be noticed that the numerical plate modelled in the present work did not respond to the flow as promptly as the physical one for $H=0.2 \mathrm{~m}$. After $t=0.31 \mathrm{~s}$, the displacement calculated by wsiFoam lags behind the experiment and Liao et al. solution. However, wsiFoam maximum displacement is similar to the experiment, whilst Liao et al. prediction is $8.3 \%$ lower. As pointed out by the authors of reference [55], the first test cases, i.e. $H=0.2 \mathrm{~m}$ and $H=0.3 \mathrm{~m}$, are more difficult to simulate due to the development of complex and turbulent flow structures as well as air cavities forming near the plate. Moreover, these authors did also consider the influence of the gate motion and, consequently, adjusted the initial problem conditions, which led to a significant improvement of their numerical results [55. However, we did not consider the influence of the gate motion in our study and, instead, modelled a perfectly steady column of water falling under gravity effects, which is common practice in the simulation of dam breaks 30 .

Figs.11] 13 show a qualitative comparison between experiments and simulations at different time instants. It can be readily seen that for $H=0.2 \mathrm{~m}$ and $t=0.27 \mathrm{~s}$ the simulated water front shape differs from the experiment probably due to the influence of the gate movement. Advancing in time, the calculated modes of deflection are in agreement with those reported in the experiment, although the maximum displacement remains underestimated except for $t \geq 0.47 \mathrm{~s}$ (see Fig. 10p. The case with $H=0.4 \mathrm{~m}$ shows the best 
(a)

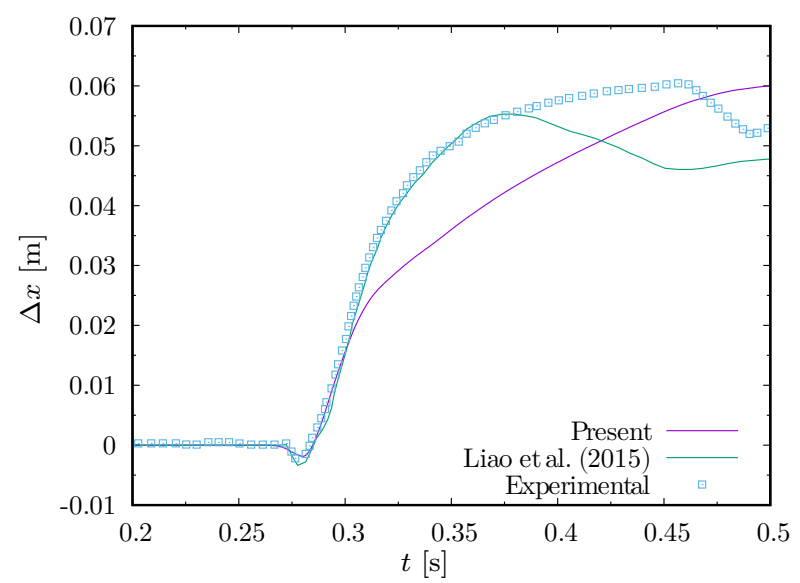

(b)

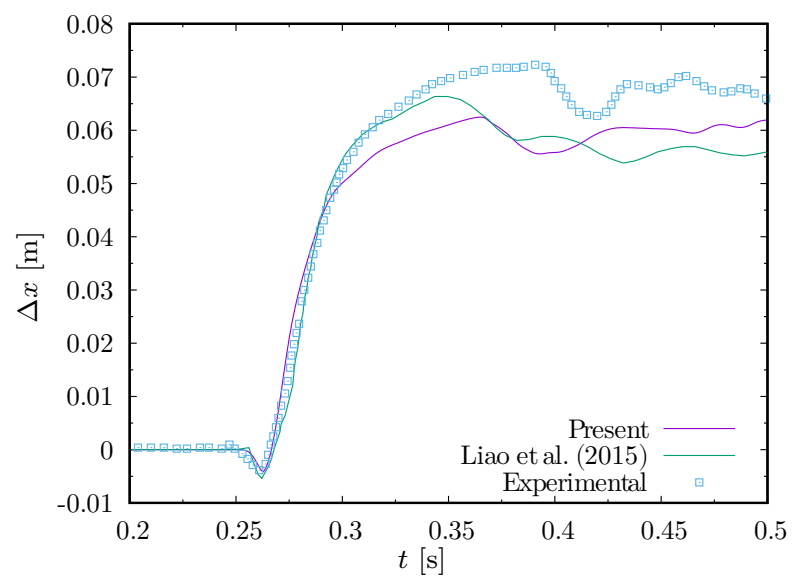

(c)

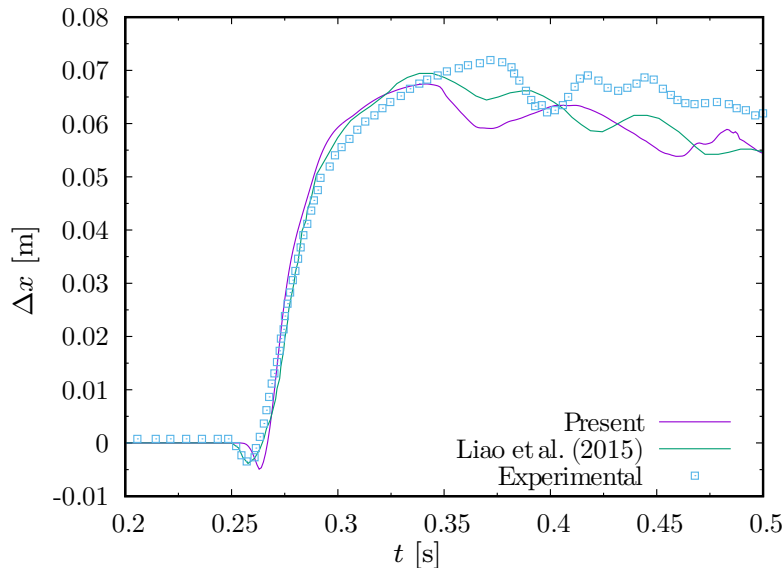

Figure 10: Water dam impact on an elastic plate: plate's tip horizontal displacement time history for (a) $H=0.2 \mathrm{~m}$, (b) $H=0.3 \mathrm{~m}$ and (c) $H=0.4 \mathrm{~m}$. 


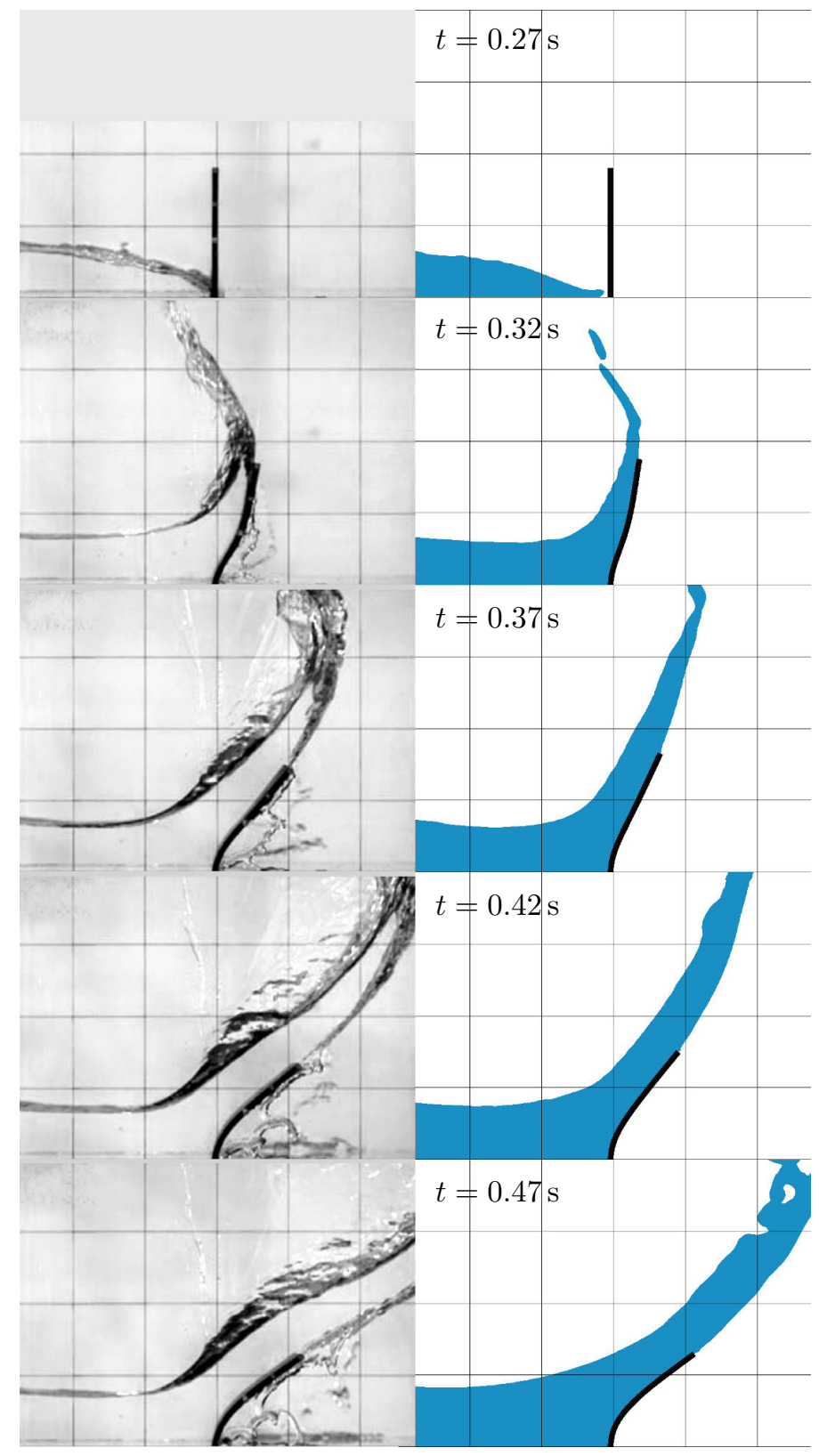

Figure 11: Water dam impact on an elastic plate: snapshots of experiments (left) and numerical simulations (right) taken at different times for $H=0.2 \mathrm{~m}$. The background squared grid is $5 \mathrm{~cm}$ long. 


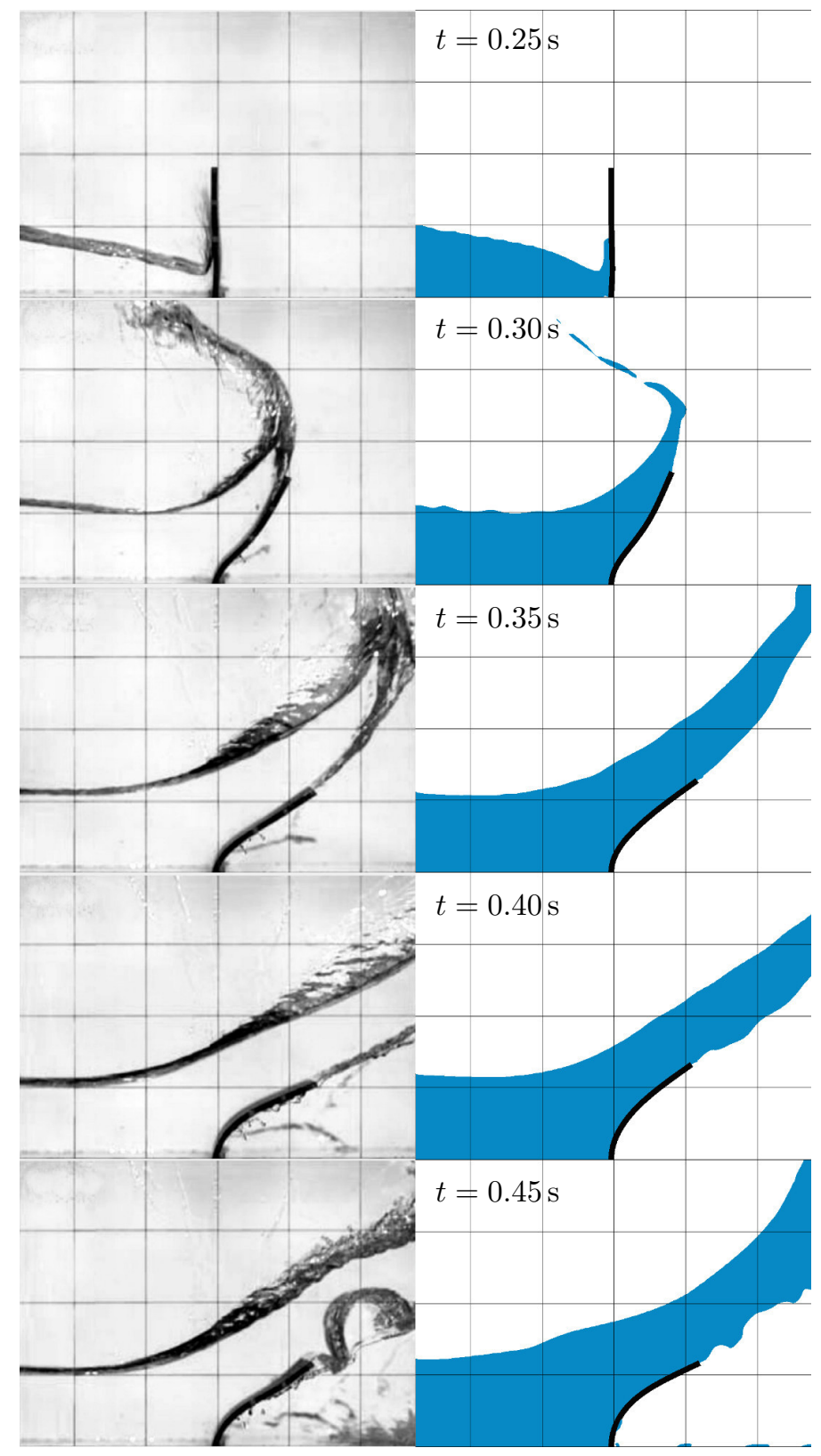

Figure 12: Water dam impact on an elastic plate: snapshots of experiments (left) and numerical simulations (right) taken at different times for $H=0.3 \mathrm{~m}$. The background squared grid is $5 \mathrm{~cm}$ long. 


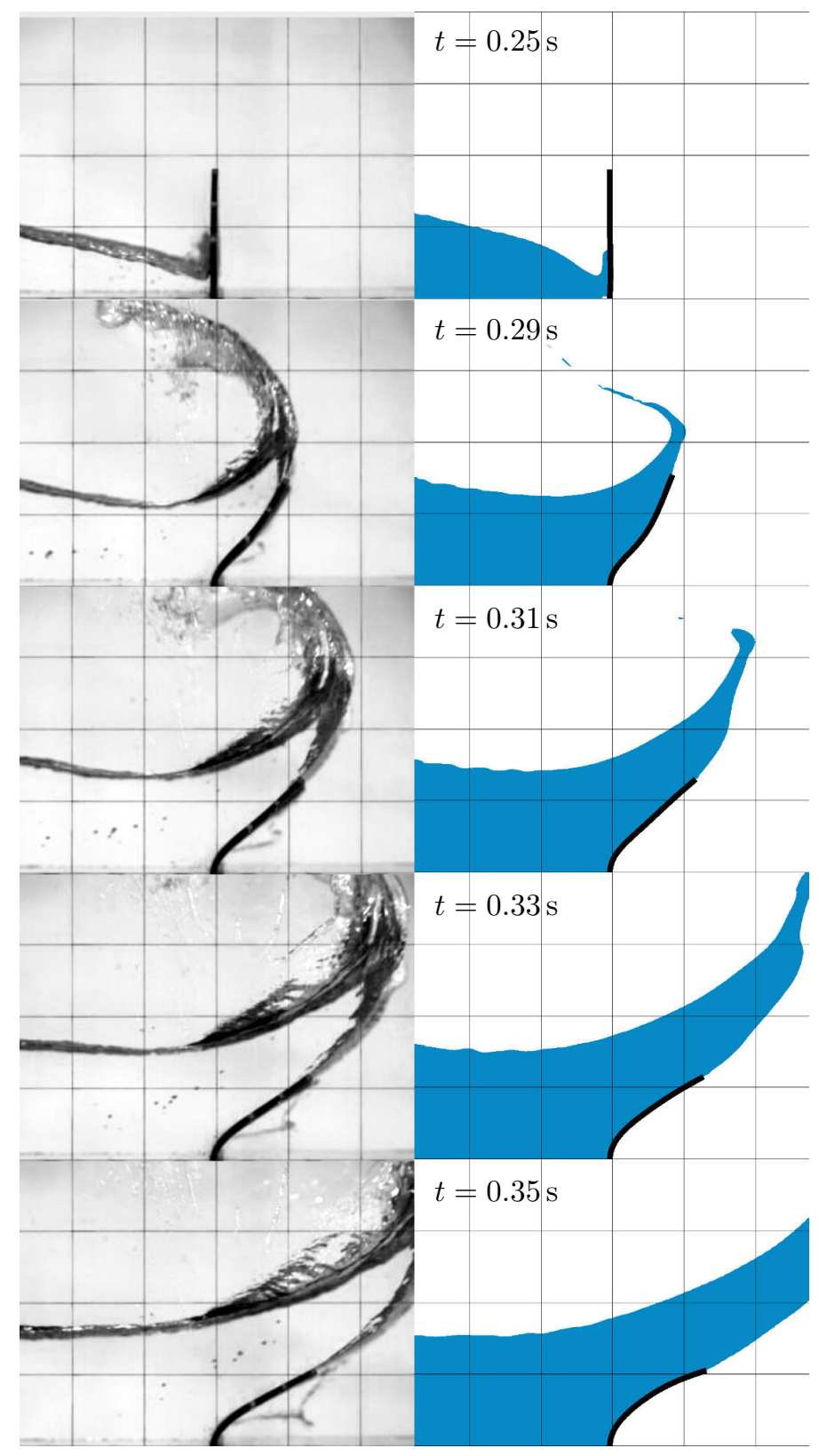

Figure 13: Water dam impact on an elastic plate: snapshots of experiments (left) and numerical simulations (right) taken at different times for $H=0.4 \mathrm{~m}$. The background squared grid is $5 \mathrm{~cm}$ long. 
Table 6: Water dam impact on an elastic plate: computation times and speed up for an increasing number of cores.

\begin{tabular}{cccccc}
\hline$H(\mathrm{~m})$ & 1 core $(\mathrm{s})$ & 2 cores $(\mathrm{s})$ & Speed up $(-)$ & 4 cores $(\mathrm{s})$ & Speed up $(-)$ \\
\hline 0.2 & 36957 & 24173 & 1.53 & 17484 & 2.11 \\
0.3 & 45373 & 28466 & 1.59 & 20887 & 2.17 \\
0.4 & 44792 & 28648 & 1.56 & 21327 & 2.10 \\
\hline
\end{tabular}

agreement between experiments and simulations and demonstrates the ability of the VOF method to track the free surface reasonably well and the FVM to handle large solid deformations.

Finally, Table 6 reports on the computation times for the three configurations and an increasing number of cores. The case with $H=0.4 \mathrm{~m}$ is the most computationally expensive due to an overall increase of the flow speed and strain experienced by the plate. In terms of parallel scalability, we get consistent speed up values slightly above 2 for $4 \mathrm{CPU}$ cores (parallel performance above $50 \%$ ), similarly to what it is obtained in Section 3.3

\section{Conclusions}

This work has introduced an accurate and efficient FSI framework based on the FVM that uses the open-source CFD library OpenFOAM. The originality of the present framework resides in the multi-region approach developed in companion articles where different regions of the computational domain, e.g. fluids and solids, can be specified and coupled whilst taking advantage of parallel domain decomposition algorithms. The CFD solver uses the VOF method with the MULES procedure to guarantee a sharp interface between the liquid and gas phases. The CSM solver uses the total Lagrangian formulation to calculate the total displacement of an isothermal, non-linear elastic solid. Finally, a partitioned approach using Aitken's adaptive relaxation coefficients allows for one- or two-way coupling between the CFD and CSM solvers.

The present method has been applied to four different benchmarks and validated against theoretical, experimental and other numerical data. The first test case has demonstrated the potential of the FVM for the simulation of elastic bodies subject to large deformations. The second benchmark proves that the present numerical framework can be applied to more simple, single-phase fluid flow FSI problems. Two additional test cases consisting of water impacts on elastic bodies have also been conducted and results compared against previous data with overall good results. The main differences observed in the present work have been attributed to the treatment of boundary conditions (Section 3.2, , usage of simplified hypothesis (Section 3.3) or the intrinsic complexity of some experiments (Section 3.4).

Close attention has been paid to the computational efficiency of the FSI framework. It has been shown 
that the total Lagrangian formulation is computationally faster than the updated Lagrangian formulation, especially during the simulation of static equilibrium problems. Another important contribution of this work has been to demonstrate that, in cases where the solid strain remains small, the proposed one-way FSI procedure gives similar results to the two-way counterpart whilst being about an order of magnitude faster. Furthermore, computational times can be significantly reduced by increasing the number of CPU cores and much better parallel performance can be expected for large-scale simulations, which renders this novel FSI method an attractive open-source candidate for the simulation of wave-structure interaction problems typically found in ocean and coastal engineering disciplines. Indeed, being OpenFOAM a CFD library widely used in industry and research, this procedure can become a popular choice among colleagues from these sectors.

Future work will focus on integrating a compressible two-phase flow solver within the present FSI framework in order to study the combined effects of hydroelasticity and compressibility that might be associated with violent wave impacts against flexible bodies, such as those occurring in sloshing tanks. Furthermore, the combination of FSI simulations together with the accurate wave modelling presented in our companion article 31] will allow us to include in the study much more complex problems in ocean, coastal and naval engineering.

\section{Acknowledgements}

The authors acknowledge with gratitude the financial support from the UK Engineering and Physical Sciences Research Council (EPSRC) under the Software for the Future (SoFT) initiative and related research grants EP/K037889/1, EP/K038168/1 and EP/K038303/1, EP/M022382/1 and EP/N008839/1. The authors also benefited from the computational resources provided by the Barcelona Supercomputing Center (BSC).

\section{References}

[1] The Catapult Programme: Offshore Renewables Opportunity. URL https://ore.catapult.org.uk/

[2] L. Yuanyuan, China makes a big bet on offshore wind, Renewable Energy World. URL http://www.renewableenergyworld.com/articles/2017/10/china-makes-a-big-bet-on-offshore-wind.html

[3] C. Lugni, M. Brocchini, O. M. Faltinsen, Wave impact loads: The role of the flip-through, Physics of Fluids 18 (12) (2006) 122101. doi:10.1063/1.2399077

URL https://doi.org/10.1063/1.2399077 
[4] G. Bullock, C. Obhrai, D. Peregrine, H. Bredmose, Violent breaking wave impacts. Part 1: results from large-scale regular wave tests on vertical and sloping walls, Coastal Engineering 54 (8) (2007) 602-617. doi:10.1016/j.coastaleng.2006. 12.002

URL https://doi.org/10.1016/j.coastaleng.2006.12.002

[5] H. Bredmose, D. H. Peregrine, G. N. Bullock, Violent breaking wave impacts. Part 2: modelling the effect of air, Journal of Fluid Mechanics 641 (2009) 389-430. doi:10.1017/s0022112009991571

URL http://dx.doi.org/10.1017/S0022112009991571

[6] Z. Ma, D. Causon, L. Qian, H. Gu, C. Mingham, P. J. Martínez-Ferrer, A GPU based compressible multiphase hydrocode for modelling violent hydrodynamic impact problems Computers \& Fluids 120 (2015) 1-23. doi:10.1016/j.compfluid. 2015.07 .010 URL http://dx.doi.org/10.1016/j.compfluid.2015.07.010

[7] A. Cappelli, R. Salzman, J. Wilkinson, Hydroelastic interaction of shells of revolution during water impact, AIAA Journal 6 (5) (1968) 792-797.

[8] R. Zhao, O. Faltinsen, Water entry of two-dimensional bodies Journal of Fluid Mechanics 246 (1993) 593-612. doi: $10.1017 / \mathrm{s} 002211209300028 \mathrm{x}$ URL http://dx.doi.org/10.1017/S002211209300028X

[9] C. Lugni, A. Bardazzi, O. M. Faltinsen, G. Graziani, Hydroelastic slamming response in the evolution of a flip-through event during shallow-liquid sloshing, Physics of Fluids 26 (3) (2014) 032108. doi:10.1063/1.4868878 URL https://doi.org/10.1063/1.4868878

[10] H. Rusche, Computational fluid dynamics of dispersed two-phase flows at high phase fractions, Ph.D. thesis, University of London (2002).

[11] Z. H. Ma, D. M. Causon, L. Qian, C. G. Mingham, H. B. Gu, P. J. Martínez-Ferrer, A compressible multiphase flow model for violent aerated wave impact problems, Proceedings of the Royal Society of London A: Mathematical, Physical and Engineering Sciences 470 (2014) . doi:10.1098/rspa.2014.0542 URL http://rspa.royalsocietypublishing.org/content/470/2172/20140542

[12] R. Vignjevic, J. Campbell, Review of development of the smooth particle hydrodynamics (SPH) method in: Predictive Modeling of Dynamic Processes, Springer US, 2009, pp. 367-396. doi:10.1007/978-1-4419-0727-1_20 URL https://doi.org/10.1007/978-1-4419-0727-1_20

[13] P.-M. Guilcher, G. Oger, E. Jacquin, L. Brosset, N. Grenier, D. Le Touzé, et al., Simulation of liquid impacts with a twophase parallel sph model, in: The 20th International Offshore and Polar Engineering Conference, International Society of Offshore and Polar Engineers, 2010.

[14] O. C. Zienkiewicz, R. L. Taylor, The Finite Element Method, Vol. 1 and 2, McGraw Hill, 1990.

[15] K.-J. Bathe, F. Brezzi, O. Pironneau, Computational fluid and solid mechanics, Springer, 2001.

[16] H. Jasak, H. G. Weller, Application of the finite volume method and unstructured meshes to linear elasticity 48.

[17] A. Slone, C. Bailey, M. Cross, Dynamic solid mechanics using finite volume methods Applied Mathematical Modelling 27 (2) (2003) 69-87. doi:10.1016/s0307-904x(02)00060-4 URL https://doi.org/10.1016/s0307-904x(02)00060-4

[18] G. Hou, J. Wang, A. Layton, Numerical methods for fluid-structure interaction - a review, Communications in Computational Physics 12 (02) (2012) 337-377. doi:10.4208/cicp.291210.290411s 
URL https://doi.org/10.4208/cicp.291210.290411s

[19] A. Robertson, J. Jonkman, F. Vorpahl, W. Popko, J. Qvist, L. Frøyd, et al., Offshore code comparison collaboration continuation within IEA wind task 30: phase II results regarding a floating semisubmersible wind system, in: ASME 2014 33rd International Conference on Ocean, Offshore and Arctic Engineering, American Society of Mechanical Engineers, 2014.

[20] D. E. Keyes, L. C. McInnes, C. Woodward, W. Gropp, E. Myra, M. Pernice, et al., Multiphysics simulations The International Journal of High Performance Computing Applications 27 (1) (2013) 4-83. doi:10.1177/1094342012468181 URL https://doi .org/10.1177/1094342012468181

[21] D. L. Brown, J. Bell, D. Estep, W. Gropp, B. Hendrickson, S. Keller-McNulty, et al., Applied mathematics at the U.S. Department of Energy: Past, present, and a view to the future, Tech. rep., Office of Science, U.S. Department of Energy (2008).

[22] J. Donea, S. Giuliani, J. Halleux, An arbitrary Lagrangian-Eulerian finite element method for transient dynamic fluidstructure interactions, Computer Methods in Applied Mechanics and Engineering 33 (1-3) (1982) 689-723. doi:10.1016/ $0045-7825(82) 90128-1$

URL https://doi .org/10.1016/0045-7825(82)90128-1

[23] C. A. Taylor, T. J. Hughes, C. K. Zarins, Finite element modeling of blood flow in arteries Computer Methods in Applied Mechanics and Engineering 158 (1-2) (1998) 155-196. doi:10.1016/s0045-7825(98)80008-x

URL https://doi.org/10.1016/s0045-7825(98)80008-x

[24] A. Slone, K. Pericleous, C. Bailey, M. Cross, C. Bennett, A finite volume unstructured mesh approach to dynamic fluid-structure interaction: an assessment of the challenge of predicting the onset of flutter. Applied Mathematical Modelling 28 (2) (2004) 211-239. doi:10.1016/s0307-904x(03)00142-2 URL https://doi.org/10.1016/s0307-904x(03)00142-2

[25] Z. Tukovic, H. Jasak, Updated Lagrangian finite volume solver for large deformation dynamic response of elastic body, Transactions of FAMENA 31 (2007) 55-70.

[26] A. Aksenov, A. Dyadkin, T. Luniewski, V. Pokhilko, Fluid structure interaction analysis using Abaqus and FlowVision, in: Abaqus Users' Conference, 2004.

[27] O. Aagaard, Hydroelastic analysis of flexible wedges, Master's thesis, Norwegian University of Science and Technology (2013).

[28] S. Ali, C. Habchi, S. Menanteau, T. Lemenand, J.-L. Harion, Three-dimensional numerical study of heat transfer and mixing enhancement in a circular pipe using self-sustained oscillating flexible vorticity generators Chemical Engineering Science 162 (2017) 152-174. doi:10.1016/j.ces.2016.12.039 URL https://doi.org/10.1016/j.ces.2016.12.039

[29] X. Lv, Y. Zhao, X. Huang, G. Xia, Z. Wang, An efficient parallel/unstructured-multigrid preconditioned implicit method for simulating 3d unsteady compressible flows with moving objects Journal of Computational Physics 215 (2) (2006) 661-690. doi:10.1016/j.jcp.2005.11.012

URL https://doi.org/10.1016/j.jcp.2005.11.012

[30] P. J. Martínez-Ferrer, D. Causon, L. Qian, C. Mingham, Z. Ma, A multi-region coupling scheme for compressible and incompressible flow solvers for two-phase flow in a numerical wave tank Computers \& Fluids 125 (2016) 116-129. doi: http://dx.doi.org/10.1016/j.compfluid.2015.11.005 
URL http://www.sciencedirect.com/science/article/pii/S004579301500376X

[31] P. J. Martínez-Ferrer, L. Qian, Z. Ma, D. Causon, C. Mingham, Improved numerical wave generation for modelling ocean and coastal engineering problems, Ocean Engineering 152 (2018) 257-272. doi:https://doi.org/10.1016/j.oceaneng. 2018.01 .052

URL https://linkinghub.elsevier.com/retrieve/pii/S0029801818300520

[32] H. Jasak, Error analysis and estimation for the finite volume method with applications to fluid flows, Ph.D. thesis, University of London (1996).

[33] N. G. Jacobsen, M. R. van Gent, G. Wolters, Numerical analysis of the interaction of irregular waves with two dimensional permeable coastal structures Coastal Engineering 102 (2015) 13-29. doi:10.1016/j.coastaleng.2015.05.004 URL https://doi.org/10.1016/j.coastaleng.2015.05.004

[34] Z. Ma, D. Causon, L. Qian, C. Mingham, P. J. Martínez-Ferrer, Numerical investigation of air enclosed wave impacts in a depressurised tank, Ocean Engineering 123 (2016) 15-27. doi:10.1016/j.oceaneng.2016.06.044 URL http://dx.doi.org/10.1016/j.oceaneng.2016.06.044

[35] H. Weller, Derivation, modelling and solution of the conditionally averaged two-phase flow equations, Nabla Ltd, No Technical Report TR/HGW/02 (2002) .

[36] K. Kissling, J. Springer, J. Jasak, S. Schutz, K. Urban, M. Piesche, A coupled pressure based solution algorithm based on the volume-of-fluid approach for two or more immiscible fluids, in: Proceedings of the V European Conference on Computational Fluid Dynamics, ECCOMAS CFD, 2010.

[37] R. Issa, Solution of the implicitly discretised fluid flow equations by operator-splitting, Journal of Computational Physics 62 (1986) 40-65. doi:10.1016/0021-9991(86)90099-9 URL http://dx.doi.org/10.1016/0021-9991(86)90099-9

[38] G. P. Galdi, R. Rannacher, Fundamental trends in fluid-structure interaction, Vol. 1, World Scientific, 2010.

[39] U. Kuttler, W. A. Wall, Fixed-point fluid-structure interaction solvers with dynamic relaxation Computational Mechanics 43 (2008) 61-72. doi:10.1007/s00466-008-0255-5 URL http://dx.doi.org/10.1007/s00466-008-0255-5

[40] Y. Zhao, A. Forhad, A general method for simulation of fluid flows with moving and compliant boundaries on unstructured grids Computer Methods in Applied Mechanics and Engineering 192 (39-40) (2003) 4439-4466. doi: $10.1016 / \mathrm{s} 0045-7825(03) 00424-9$ URL https://doi.org/10.1016/s0045-7825(03)00424-9

[41] H. Jasak, Z. Tukovic, Automatic mesh motion for the unstructured finite volume method, Transactions of FAMENA 30 (2006) 1-20.

[42] K. Mattiasson, Numerical results from large deflection beam and frame problems analysed by means of elliptic integrals, International journal for numerical methods in engineering 17 (1) (1981) 145-153.

[43] J. V. Vazquez, Nonlinear analysis of orthotropic membrane and shell structures including fluid-structure interaction, $\mathrm{Ph} . \mathrm{D}$. thesis, Universitat Politecnica de Catalunya (2007).

[44] U. Küttler, C. Förster, W. A. Wall, A solution for the incompressibility dilemma in partitioned fluid-structure interaction with pure dirichlet fluid domains. Computational Mechanics 38 (4-5) (2006) 417-429. doi:10.1007/s00466-006-0066-5 URL https://doi.org/10.1007/s00466-006-0066-5

[45] C. Kassiotis, A. Ibrahimbegovic, R. Niekamp, H. G. Matthies, Nonlinear fluid-structure interaction problem. Part I: 
implicit partitioned algorithm, nonlinear stability proof and validation examples, Computational Mechanics 47 (3) (2010)

305-323. doi:10.1007/s00466-010-0545-6

URL https://doi.org/10.1007/s00466-010-0545-6

[46] C. Habchi, S. Russeil, D. Bougeard, J.-L. Harion, T. Lemenand, A. Ghanem, D. D. Valle, H. Peerhossaini, Partitioned solver for strongly coupled fluid-structure interaction Computers \& Fluids 71 (2013) 306-319. doi:10.1016/j.compfluid. 2012.11 .004

URL https://doi.org/10.1016/j.compfluid.2012.11.004

[47] D. P. Mok, Partitionierte losungsansatze in der strukturdynamik und der fluid-struktur-interaktion, Ph.D. thesis, Universitat Stuttgart (2001).

[48] C. Lu, Y. He, G. Wu, Coupled analysis of nonlinear interaction between fluid and structure during impact, Journal of Fluids and Structures 14 (2000) 127-146. doi:10.1006/jfls.1999.0257.

URL http://dx.doi.org/10.1006/jfls.1999.0257

[49] K. Liao, C. Hu, W. Duan, Two-dimensional numerical simulation of an elastic wedge water entry by a coupled FDM-FEM method, Journal of Marine Science and Application 12 (2013) 163-169. doi:10.1007/s11804-013-1181-2

URL http://dx.doi.org/10.1007/s11804-013-1181-2

[50] A. Shams, M. Porfiri, Treatment of hydroelastic impact of flexible wedges, Journal of Fluids and Structures 57 (2015) 229-246. doi:10.1016/j.jfluidstructs.2015.06.017

URL https://doi.org/10.1016/j.jfluidstructs.2015.06.017

[51] P. J. Martínez-Ferrer, D. M. Causon, L. Qian, C. G. Mingham, Z. H. Ma, Modelling wave interaction with deformable structures based on a multi-region approach within OpenFOAM, in: Proceedings of the 27th International Ocean and Polar Engineering Conference, 2017, pp. 678-685.

[52] M. Culpo, Current bottlenecks in the scalability of OpenFOAM on massively parallel clusters, PRACE paper.

[53] J. C. Martin, W. J. Moyce, Part IV. An experimental study of the collapse of liquid columns on a rigid horizontal plane, Phil. Trans. R. Soc. Lond. A 244 (1952) 312-324.

[54] Z. Ma, L. Qian, P. J. Martínez-Ferrer, D. M. Causon, C. G. Mingham, W. Bai, An overset mesh based multiphase flow solver for water entry problems Article in press in Computers \& Fluidsdoi:10.1016/j.compfluid.2018.01.025 URL https://doi.org/10.1016/j.compfluid.2018.01.025

[55] K. Liao, C. Hu, M. Sueyoshi, Free surface flow impacting on an elastic structure: Experiment versus numerical simulation. Applied Ocean Research 50 (2015) 192-208. doi:10.1016/j.apor.2015.02.002

URL http://dx.doi.org/10.1016/j.apor.2015.02.002 